\title{
Archaeobotanical investigations of Late Neolithic lakeshore settlements (Lake Biel, Switzerland)
}

\author{
C. Brombacher \\ Botanisches Institut der Universität Basel, Schönbeinstrasse 6, CH-4056 Basel, Switzerland
}

Received February 3, 1997 / Accepted April 15, 1997

\begin{abstract}
This paper describes the results of the archaeobotanical examination of four Late Neolithic lakeshore settlements on Lake Biel in Switzerland. Due to the excellent preservation conditions in lakeshore settlements, non-carbonized as well as carbonized seeds and fruits were recovered in large numbers. In addition to the diaspore analyses, some samples of charcoal and moss were identified. The spectrum of cultivated plants was markedly different between the sites. In the transition from the 34 th to 33 rd century B.C. (sites Nidau, Lüscherz and Lattrigen 'VI') naked wheat and barley were predominant, while in the 32 nd century B.C. (Lattrigen 'VII') emmer (a glume wheat) was most abundant. Flax and opium poppy were of great importance during the whole late Neolithic period. In addition to cereals, a large number of cereal weeds were detected. The wild flora included a high percentage of aquatic and lake-shore plants which results from the strong influence of water on the cultural layers. Taxa of flood-plain forest are also common. The proportion of potential grassland plants was low (in total only 13 taxa) which suggests that in addition to the cultivated fields only few pastures and grassland areas existed close to the settlements.
\end{abstract}

Key words: Plant macrofossils - Lakeshore settlements - Late Neolithic - Cultivated plants - Switzerland

\section{Introduction}

Lake Biel is of glacial origin and is situated in the Alpine foothills (Alpenvorland) in western Switzerland south-east of the Jura mountains at ca. $430 \mathrm{~m}$ asl (Fig. 1). The area was covered during the upper Würmian by the most recent advance of the Rhone glacier. The north shore of the lake has relatively steep banks which are part of the southern edge of the Jura mountains, and in just $8 \mathrm{~km}$ they climb to $1600 \mathrm{~m}$ asl. The south facing side borders on the Swiss plateau (the relatively flat lowlands between the Jura mountains and the Alps) and has quite flat banks. In accordance with the topographical situation, the Jura bank is only covered in certain places by a narrow alluvial zone. On the steep-sloped limestone

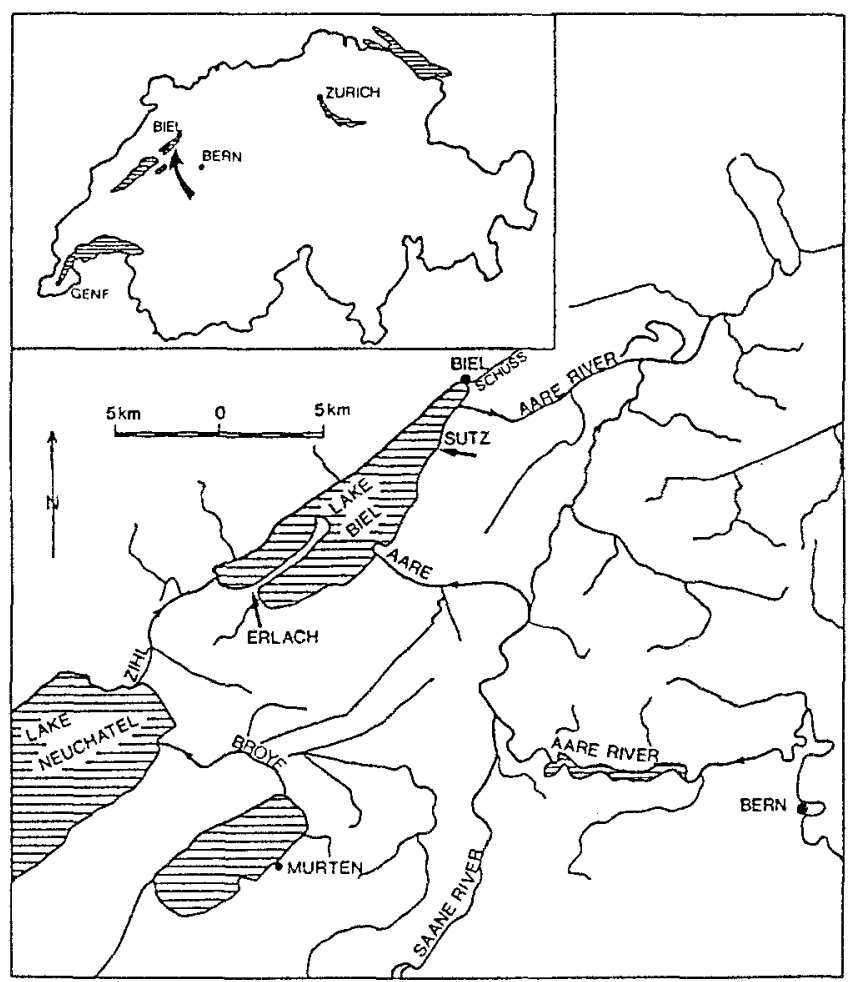

Fig. 1. Situation of Lake Biel in western Switzerland (after Wohlfarth and Schneider 1991)

above the banks, mainly xerothermic mixed oak woods with Quercus pubescens grow, and on flat ground, natural arid grasslands (Xerobromion) occur, vegetation types which are protected (Hegg et al. 1993). These dry places are today largely occupied by vineyards. The opposite, flat bank is a very different natural habitat; on the low lying ground close to the lake with its underlying geology of moraines, river and lake beds, and peat, large areas are dominated by successions starting from open, still water or by fen woods and flood plains (AmmannMoser 1975). Forests of deciduous hardwoods composed of members of the Fagaceae, mainly beech (Fagus sylvatica), are the natural vegetation of the slightly higher lying Molasse areas (see Hegg 1980). 


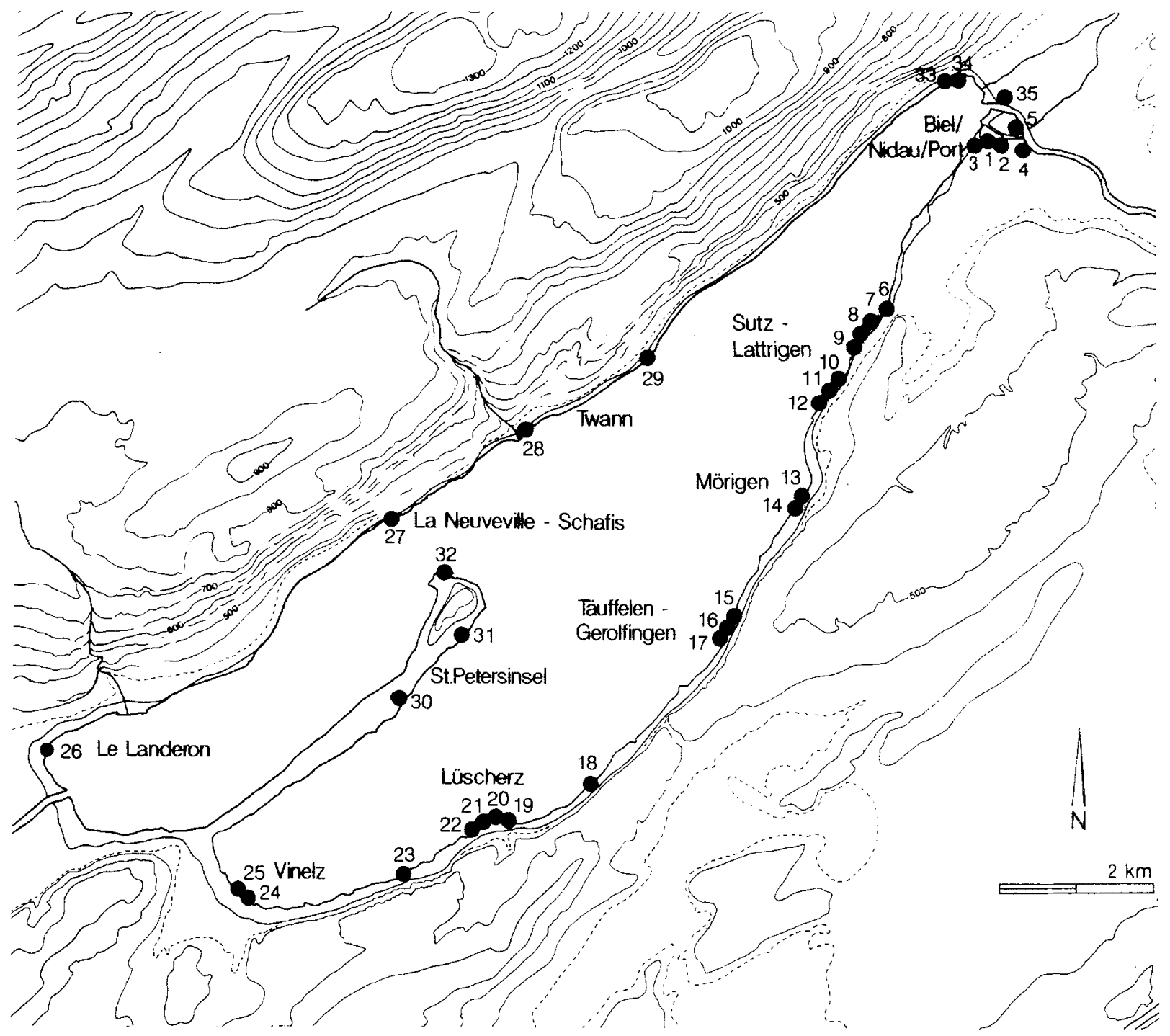

Fig. 2. Map of the excavation sites and settlement periods (after Hafner 1996)

It was known as early as the last century that there were prehistoric lakeshore settlements on Lake Biel. The discovery of pile dwellings on lakes in Canton Zürich in the middle of the last century and the pile dwelling theory of Keller (1854) led to a collecting boom at Lake Biel, as a result of which the building structures and layers of many sites were carelessly destroyed and the archaeological material was plundered.

An artificial lowering of the lake level by about $2 \mathrm{~m}$ and the construction of an intensive drainage network in 1888-1892 and 1962-1973 changed the hydrology and the lake shore vegetation to a great extent. This caused a continuous loss of cultural deposits from the prehistoric sites, which during the last decades has been intensified by boat traffic and other leisure activities.

In order to determine the degree of erosion impact, the Archaeological Service of Canton Bern decided to undertake an inventory of the Neolithic and Bronze Age lakeshore settlements around lake Biel: this took place between 1984 and 1987. In addition to examination of the old sites, drilling cores and underwater samples were also taken (Winiger 1989) and pollen analyses of the
Late Glacial and early Holocene periods were carried out (Wohlfarth and Schneider 1991). In total, ten settlement concentrations were located which were mainly situated on the southern shore (Fig. 2). All the sites had been occupied several times and are dendrochronologically dated. The Neolithic settlement period was from ca. 3840 B.C. -2600 B.C., shorter periods in the early Bronze Age (ca. 1630-1570 B.C.) and late Bronze Age (ca. 1050-850 B.C.) have also been documented. On the basis of these results, several rescue excavations have been performed in recent years.

Macrobotanical remains have been examined to date from the four Late Neolithic sites, dated between 3400 and ca. 3000 B.C. (Fig. 2). One of these sites, NidauSchlossmatte/BKW 1991 (Ib), layer 5, ca. 3410-3380 cal. B.C., is situated at the efflux of the lake in a floodplain forested area. It was examined in a rescue excavation in 1991 (excavation area ca. $600 \mathrm{~m}^{2}$ ). The other three stations: Sutz-Lattrigen - Lattrigen Riedstation (VI), 3393-3388 B.C.; Sutz-Lattrigen - Lattrigen Hauptstation, aussen (VII), 3202-3013 B.C.; and LüscherzKleine Station (XV), 3403-3386 B.C., are situated on the 

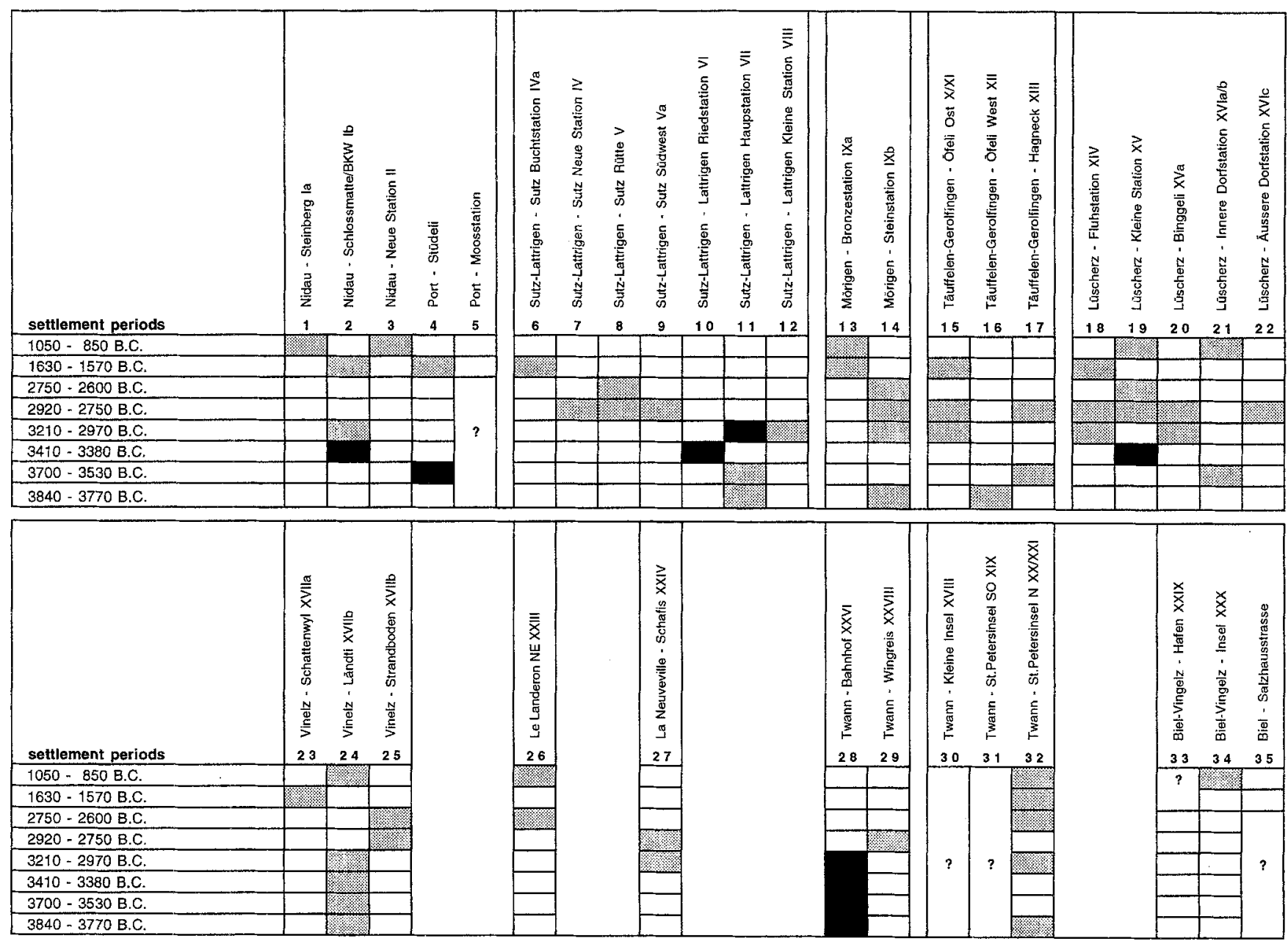

after Hafner (1996)

archaeobotanically investigated (Port-Stüdeli (4): Jacomet unpubl.)

Legend to Fig. 2

right flat bank of the lake. The cultural layers at these sites lie in the lake below today's water level. In Lattrigen, large underwater excavations have been carried out since 1988: in the winter months of 1988-1990 a $6000 \mathrm{~m}^{2}$ excavation area of Site 'VI' (Lattrigen-Riedstation) was examined, and between 1988 to 1992 a preliminary survey was carried out of Late Neolithic layers of Site 'VII' (Hauptstation-aussen). Since the beginning of 1996, large scale rescue excavations have also been carried out at this site. In Lüscherz 'Kleine Station' as part of an inventory, an underwater survey was performed in 1987 which yielded a few botanical samples (Winiger 1989).

The degree to which the archaeobotanical examinations have been completed at the individual sites varies. The best studied is Lattrigen 'VII' from where the most samples (69) originate. At this site, the layers representing two archaeological phases could be clearly differentiated. The lower, better preserved group of layers includes cultural layers 1-3 and is dendrochronologically dated to 3203-3139 B.C. In contrast, the upper, later layer which corresponds to the second archaeological phase, layer 0 (dated 31 st century B.C.) was more heavily eroded and could only be followed over a small part of the examined area.

The Nidau site ( 30 samples) can also be classed as well examined, although the cultural layers were strongly affected by water and flooded. Several cultural layers could be distinguished of which only the deepest, layer 5 , that corresponds to the settlement period of 3400 . B.C. (3403-3386), was examined in detail. This layer was of only limited thickness $(5-10 \mathrm{~cm})$ and remained in situ in only part of the excavation area. Before the next settlement period in the 32 nd century B.C., which is represented by the only slightly organically coloured layer 3, a part of the settlement area was covered by an ancient branch of the efflux of lake Biel. As a result the material of layer 5 was washed up and deposited in a secondary position. Only limited comparisons can be made with Lattrigen 'VI' where the cultural layer was completely eroded and Lüscherz 'Kleine Station'. From these two sites only seven judgement samples (after van der Veen 1987; Jones 1988) were available. 
The first comprehensive archaeobotanical investigations at Lake Biel were carried out on the material collected from site Twann which was excavated in 19741976. Large sample volumes of the cultural layers of the Cortaillod Culture (37/36th century B.C.) and Late Neolithic were studied for their macrofossils (Bollinger and Jacomet 1981; Piening 1981). These serve as a basic comparison for the investigation described here.

\section{Material and Methods}

From the four sites, a total of 106 botanical samples were available (Table 1). They originated in Nidau 'Schlossmatte/ $\mathrm{BKW}^{\prime}$ (30 samples with a total volume of 24.5 1), Lattrigen 'VII' (69 samples, total volume 29.6 1), Lattrigen 'VI' (2 samples, total volume 0.11 ), and Lüscherz 'Kleine Station' (5 samples, total volume 0.51 ). The sampling method for the two intensively sampled sites, Nidau 'BKW' and Lattrigen 'VII', was developed from the experience derived from the lakeshore settlements in Zürich (Jacomet et al. 1989); both interval samples from each layer (horizontal distance between samples $1 \mathrm{~m}$ to several $\mathrm{m}$ ) and judgement samples visibly rich in plant material (such as cereal and moss samples) were taken. The volume of the interval samples was between 400 and $2500 \mathrm{ml}$ (average $950 \mathrm{ml}$ ), and that of the selected samples between 1 and $150 \mathrm{ml}$. Only judgement samples (stored grain) were available from Lattrigen 'VI' and Lüscherz. The sampling system at Nidau and Lattrigen 'VII' was in principle identical, but was modified according to the excavation technique. In Nidau, the cultural layer was broken down using the strip-excavation technique both above and below water so that samples could be taken every $2-5 \mathrm{~m}$ from the cross and longitudinal sections. The area, which was excavated in 1991, was $600 \mathrm{~m}^{2}$. The archaeobotanical investigation was confined to the better preserved cultural layer 5 . In Lattrigen 'VII', a quadrat of $100 \mathrm{~m}^{2}$ in the middle of the settlement and extending from it, four $2 \mathrm{~m}$ wide and $77 \mathrm{~m}$ long sample transects were examined in an underwater excavation (total area examined $730 \mathrm{~m}^{2}$, with a sample distance of $1-3 \mathrm{~m}$ in the middle and about $10 \mathrm{~m}$ in the transect area). Material from two occupation phases could be recovered: from the period 3202-3139 B.C. (layers 1-3), and from the 31 st century B.C. (layer 0). The cultural layers in Lüscherz were also underwater, the samples being collected during an underwater survey which was carried out in 1987.

The waterlogged sample material analysed was mainly from organic cultural layers, which experience showed to contain most plant material. A few samples were from the contact zone with lacustrine chalk and mixed sandy sediments that lay under and over, respectively, the cultural layers.

The samples were fine sieved with meshes of $8,4,2,1$, 0.5 , and $0.25 \mathrm{~mm}$. Charcoal isolated from the 8 and $4 \mathrm{~mm}$ fractions and seeds and fruits from all the fractions were examined. A maximum of $50 \mathrm{ml}$ of the $0.5 \mathrm{~mm}$ fraction and $10 \mathrm{ml}$ of the $0.25 \mathrm{~mm}$ fraction were examined, and the number of seeds and fruits of each taxon in these subsamples was used to derive the number for the whole sample.

\section{Results and discussion}

Density (Fig. 3)

Generally, the density of grains and fruits in cultural layers of lake shore settlements is very high. From the intensively studied lake shore settlements in Zürich
(Jacomet et al. 1989) the better preserved layers of organic sediments yielded on average 1000-5000 items per litre. On comparing judgement samples, the variation is of course higher.

Of the sites described here, the density at Lattrigen 'VII', with averages of 650-2000 in three of the four layers, is significantly higher than in Nidau, which can be accounted for by the better preservation conditions. The frequency distribution for samples has a maximum of 2000 items/l. In Nidau, where the average is only 900 items/ 1 , most of the samples contain between 500 and 1000 items $/ 1$.

\section{Presence of plant remains}

Considering the two most intensively examined sites, Nidau and Lattrigen 'VII', 36 of about 160 identified taxa appear in more than $50 \%$ of the samples, but only a few, however, reach a presence of $90 \%$. At both sites, these are Papaver somniferum, Fragaria vesca, and Rubus fruticosus. In Nidau, Schoenoplectus lacustris, and in Lattrigen 'VII', Linum usitatissimum, Malus sylvestris and Najas marina were also found in $90 \%$ of the samples. Also appearing with high presence $(>80 \%)$ at least at one site were Abies alba (needles), Hordeum vulgare (carbonized grains), Physalis alkekengi, Rosa spp. and Triticum dicoccon (uncarbonized spikelet forks). All of these taxa are exclusively ones that either arrived at the settlement through human activities (cultivated and edible/useful plants) or were washed up from the water or lake shore.

\section{Crop plants}

Altogether, seven different cultivated plants could be detected. The large amounts of carbonized cereal grains indicate that cereals played an important role in the nutrition of the Late Neolithic population. The three most important cereals are Hordeum vulgare, Triticum dicoccon and T. aestivum/durum/turgidum (Fig. 4). The most frequently identified is barley $(H$. vulgare) with 8833 grains. From Lattrigen 'VII', even whole or broken ear fragments (52 specimens) were found (Figs. 7, 9). Several samples from Nidau and Lüscherz contained baked grains, which made their identification difficult. The proportion of barley in the cereal spectrum varies between $38 \%$ in Lattrigen and $74 \%$ in Nidau. As our previous investigations of lake shore settlements showed (Jacomet et al. 1989), chaff fragments of barley are much rarer than grains. In total, only 231 carbonized and 7 uncarbonized rachis segments were found.

Of the wheats, naked wheat (Triticum aestivum/ durum/turgidum) mainly of tetraploid type and emmer (T. dicoccon), a glume wheat, were the most important. However, the number of finds of the two different wheat types at the sites differs markedly. From around 3400 B.C., finds of naked wheat are more numerous. From Nidau, 1042, from Lattrigen 'VI' 23 and from Lüscherz, 2960 grains could be identified. There are clear differences in the spectrum of wheats at Lattrigen 'VII' (ca. 3200-3000 B.C.). Here, emmer (T. dicoccon) is the most common wheat, represented by 586 grains, while naked 
Table 1. Neolithic plant remains: Lake Biel

Site

Feature (cultural layer)

Dating (B.C.)

Number of samples

Total volume in litres
Nidau 'BKW'

$\begin{array}{cc}\text { NID5 } & \text { NID5 } \\ 3400 & 3400 \\ 30 & 30 \\ 24.52 & 23.57\end{array}$

number all samples presence interval samples
Lüscherz Lattrigen 'VI' Lattrigen 'VII'

$\begin{array}{cc}\text { LUS } & \text { LAT } \\ 3400 & 3400 \\ 5 & 2 \\ 0.05 & 0.01\end{array}$

LAT0-3

LAT0-3

$3200-3050 \quad 3200-3050$

$69 \quad 41$

$29.68 \quad 28.76$

number number number presence all samples all samples all samples interval

Uncarbonized plant remains

\author{
cultivated plants \\ Anethum graveolens \\ Apium graveolens \\ Cerealia \\ Cerealia \\ Hordeum vulgare \\ Linum usitatissimum \\ Linum usitatissimum \\ Papaver somniferum \\ Pisum sativum \\ Triticum aestivum/durum \\ Triticum dicoccon \\ Triticum monococcum \\ Triticum monococcum/dicoccon \\ Triticum spec. \\ segetal weeds \\ Aethusa cynapium \\ Brassica rapa \\ Capsella bursa-pastoris \\ Chenopodium polyspermum \\ Polygonum persicaria \\ Solanum nigrum \\ Stellaria media \\ Aphanes arvensis \\ Camelina sativa \\ Campanula rapunculoides \\ Fallopia convolvulus \\ Silene cretica \\ Valerianella dentata \\ Valerianella locusta \\ Valerianella rimosa \\ Viola tricolor \\ ruderal weeds \\ Arctium cf. minus \\ Arenaria serpyllifolia \\ Barbarea vulgaris \\ Carex hirta (-type) \\ Chenopodium album \\ Chenopodium ficifolium \\ Chenopodium spec. \\ Cirsium arvense \\ Cirsium vulgare \\ Daucus carota \\ Galeopsis tetrahit \\ Hyoscyamus niger \\ Lamium of. maculatum \\ Lapsana communis \\ Linaria vulgaris
}

seed/fruit

seed/fruit

chaff

pericarp

rachis segments

capsule segments

seed/fruit

seed/fruit

seed/fruit

rachis segments

spikelet forks

spikelet forks

spikelet forks

rachis segments

$\begin{array}{rr}1 & 5.3 \\ 4 & 15.8 \\ 4 & 5.3 \\ 12 & 21.1 \\ - & - \\ 1031 & 84.2 \\ 1670 & 89.5 \\ 2105 & 94.7 \\ 1 & 5.3 \\ 5 & 5.3 \\ - & - \\ - & - \\ - & - \\ 6 & 10.5\end{array}$

seed/fruit

seed/fruit

seed/fruit

seed/fruit

seed/fruit

seed/fruit

seed/fruit

seed/fruit

seed/fruit

seed/fruit

seed/fruit

seed/fruit

seed/fruit

seed/fruit

seed/fruit

seed/fruit

seed/fruit

seed/fruit

seed/fruit

seed/fruit

seed/fruit

seed/fruit

seed/fruit

seed/fruit

seed/fruit

seed/fruit

seed/fruit

seed/fruit

seed/fruit

seed/fruit seed/fruit

$\begin{array}{rr}6 & 9.7 \\ 1 & 2.4 \\ - & - \\ 41 & 14.6 \\ 7 & 14.6 \\ 1944 & 92.7 \\ 4659 & 97.6 \\ 12617 & 100.0 \\ - & - \\ 11 & 14.6 \\ 6353 & 80.5 \\ 2 & 2.4 \\ 1571 & 17.0 \\ - & -\end{array}$

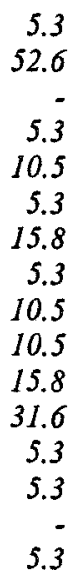

10
17
2
12
52
3
39
39
12
9
105
45
20

1
7

19.5

24.4

2.4

19.5

53.6

7.3

48.7

51.2

21.9

21.9

70.7

53.6

29.2

2.4

14.6

$\begin{array}{rr}212 & 68.3 \\ 10 & 12.2 \\ 2 & 4.8 \\ 8 & 12.2 \\ 122 & 58.5 \\ - & . \\ 4 & 9.7 \\ - & - \\ 4 & 2.4 \\ 12 & 24.4 \\ 8 & 14.6 \\ 1 & 2.4 \\ - & - \\ 89 & 73.1 \\ 2 & 2.4\end{array}$


Site

Feature (cultural layer)

Dating (B.C.)

Number of samples

Total volume in litres
Nidau 'BKW'

$\begin{array}{cc}\text { NID5 } & \text { NID5 } \\ 3400 & 3400 \\ 30 & 30 \\ 24.52 & 23.57 \\ & \\ \text { number } & \begin{array}{c}\text { presence } \\ \text { interval } \\ \text { all samples } \\ \text { samples }\end{array}\end{array}$

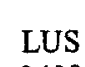

Lattrigen 'VI'

LUS
3400
5
0.05

LAT

3400

2
0.01

number

number number

all samples all samples
Lattrigen 'VII'

LAT0-3 LAT0-3

3200-3050 3200-3050

29.68

28.76 number presence

interval

samples

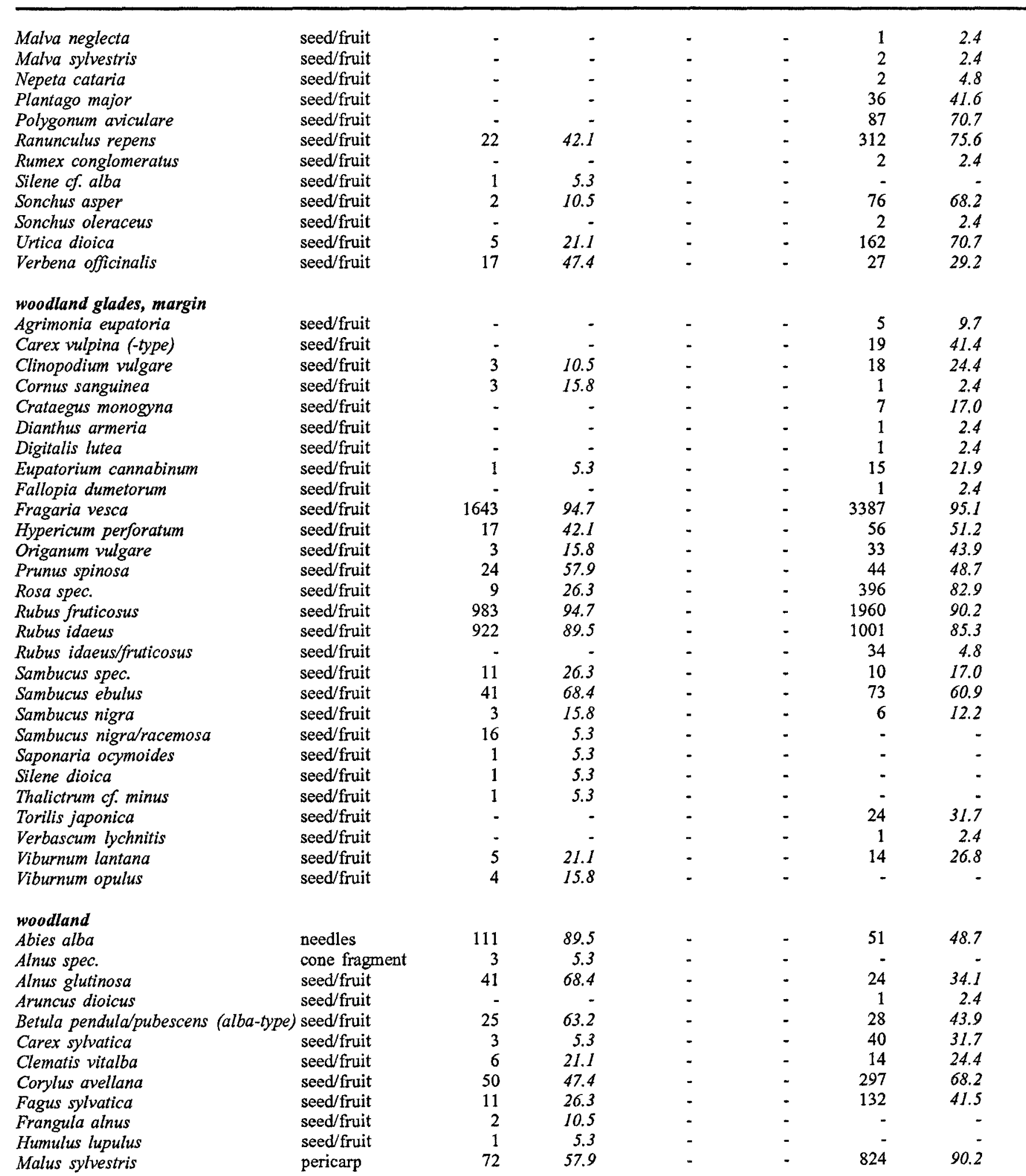


Site

Feature (cultural layer)

Dating (B.C.)

Number of samples

Total volume in litres
Nidau 'BKW'

NID5

3400

30

24.52

3400

30

23.57

number presence

all samples interval

samples
Lüscherz Lattrigen 'VI'

Lattrigen 'VII'

$\begin{array}{cccc}\text { LUS } & \text { LAT } & \text { LAT0-3 } & \text { LAT0-3 } \\ 3400 & 3400 & 3200-3050 & 3200-3050 \\ 5 & 2 & 69 & 41 \\ 0.05 & 0.01 & 29.68 & 28.76\end{array}$

number number number presence all samples all samples all samples interval

samples

\begin{tabular}{|c|c|c|c|c|c|c|c|}
\hline Malus sylvestris & seed/fruit & 28 & 57.9 & - & - & 414 & 73.1 \\
\hline Moehringia trinervia & seed/fruit & 16 & 57.9 & - & - & 72 & 56.1 \\
\hline Physalis alkekengi & seed/fruit & 391 & 89.5 & - & - & 100 & 43.5 \\
\hline cf. Phyteuma spicatum & seed/fruit & - & 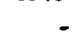 & - & - & 3 & 7.3 \\
\hline Picea abies & wing fragment & 1 & 5.3 & - & - & - & 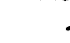 \\
\hline Poa cf. nemoralis & seed/fruit & - & - & - & - & 1 & 2.4 \\
\hline Prunus padus & seed/fruit & 5 & 15.8 & - & - & 5 & 12.2 \\
\hline Pteridium aquilinum & leaf & - & - & - & - & 11 & 19.5 \\
\hline Quercus spec. & seed/fruit & 14 & 36.8 & - & - & 294 & 75.0 \\
\hline Rubus caesius & seed/fruit & 8 & 36.8 & - & - & 26 & 39.6 \\
\hline Rumex sanguineus & seed/fruit & - & - & - & - & 25 & 14.0 \\
\hline Scrophularia of. nodosa & seed/fruit & 2 & 10.5 & - & - & 8 & 17.0 \\
\hline Stachys of. syluatica & seed/fruit & 2 & 10.5 & - & - & - & . \\
\hline Teucrium scorodonia & seed/fruit & . & - & - & - & 11 & 21.5 \\
\hline Tilia cf. platyphyllos & seed/fruit & 3 & 5.3 & - & - & - & 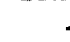 \\
\hline Viola reichenbachiana-type & seed/fruit & 3 & 15.8 & - & - & 3 & 7.3 \\
\hline Viscum album & leaf & 9 & 5.3 & - & - & 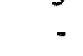 & . \\
\hline \multicolumn{8}{|l|}{ pasture, grassland } \\
\hline Agrostis spec. & seed/fruit & - & - & - & - & 1 & 2.4 \\
\hline Ajuga reptans & seed/fruit & 4 & 21.1 & - & - & 80 & 48.8 \\
\hline Alchemilla vulgaris & seed/fruit & - & 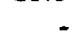 & - & - & 1 & 2,4 \\
\hline Campanula glomerata & seed/fruit & - & - & - & - & 2 & 4.8 \\
\hline Cerastium fontanum & seed/fruit & 3 & 15.8 & - & - & 209 & 75.0 \\
\hline Deschampsia cf. caespitosa & seed/fruit & - & 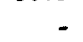 & - & - & 1 & 2.4 \\
\hline Gentiana cruciata & seed/fruit & - & - & - & - & 1 & 2.4 \\
\hline Luzula multiflora & seed/fruit & - & - & - & - & 10 & 21.5 \\
\hline Potentilla reptans & seed/fruit & 2 & 10.5 & - & - & 2 & 4.8 \\
\hline Prunella vilgaris & seed/fruit & - & - & - & - & 62 & 56.1 \\
\hline Stellaria graminea & seed/fruit & - & - & - & - & 35 & 41.4 \\
\hline Taraxacum officinale & seed/fruit & - & - & - & - & 1 & 2.4 \\
\hline Trifolium spec. & seed/fruit & 1 & 5.3 & - & - & 4 & 9.7 \\
\hline \multicolumn{8}{|l|}{ aquatic, lakreshore } \\
\hline Alisma spec. & seed/fruit & 2 & 10.5 & - & - & 2 & 2.4 \\
\hline Carex elata/gracilis & seed/fruit & - & - & - & - & 26 & 31.7 \\
\hline Chara div. spec. & oogonia & 11170 & 84.2 & - & - & 83 & 60.9 \\
\hline Corrigiola litoralis & seed/fruit & 1 & 5.3 & - & - & - & . \\
\hline Cyperus fuscus & seed/fruit & 4 & 21.1 & - & - & 5 & 9.7 \\
\hline Epilobium hirsutum & seed/fruit & - & - & - & - & 4 & 9.7 \\
\hline Linum catharticum & seed/fruit & - & - & - & - & 2 & 4.8 \\
\hline Lycopus europaeus & seed/fruit & 42 & 63.2 & - & - & 9 & 14.0 \\
\hline Mentha aquatica/arvensis & seed/fruit & 19 & 36.8 & - & - & 9 & 19.5 \\
\hline Molinia caerulea & seed/fruit & 1 & 5.3 & - & - & 18 & 34.1 \\
\hline Myosoton aquaticum & seed/fruit & 3 & 15.8 & - & - & 29 & 29.2 \\
\hline Myriophyllum spicatum & seed/fruit & 105 & 73.7 & - & - & . & . \\
\hline Najas cf. intermedia & seed/fruit & 1 & 5.3 & - & - & . & . \\
\hline Najas flexilis & seed/fruit & 1 & 5.3 & - & - & 36 & 39.0 \\
\hline Najas marina & seed/fruit & 6 & 10.5 & - & - & 381 & 92.0 \\
\hline Nasturtium officinale & seed/fruit & - & - & - & - & 1 & 2.4 \\
\hline Nitella spec. & oogonia & 15 & 10.5 & - & - & 3 & 2.4 \\
\hline Nuphar lutea & seed/fruit & 54 & 52.6 & - & - & . & . \\
\hline Nymphaea alba & seed/fruit & 1 & 5.3 & - & - & - & . \\
\hline Phragmites australis & seed/fruit & 9 & 36.8 & - & - & 3 & 7.3 \\
\hline Poa cf. palustris & seed/fruit & - & - & - & - & 2 & 4.8 \\
\hline Polygonum hydropiper & seed/fruit & 2 & 10.5 & - & - & 21 & 29.2 \\
\hline
\end{tabular}


Site

Feature (cultural layer)

Dating (B.C.)

Number of samples

Total volume in litres
Nidau 'BKW'

$\begin{array}{cc}\text { NID5 } & \text { NID5 } \\ 3400 & 3400 \\ 30 & 30 \\ 24.52 & 23.57\end{array}$

number all samples

presence

interval
Polygonum minus

Polygonum mite

Potamogeton spec.

Potamogeton cf. natans

Potamogeton pectinatus

Potamogeton perfoliatus

Potentilla supina

Ranunculus aquatilis

Ranunculus sceleratus

Schoenoplectus spec.

Schoenoplectus lacustris

Typha latifolia

Zannichellia palustris

\section{various}

Apiaceae (Umbelliferae)

Asteraceae (Compositae)

Brassicaceae (Cruciferae)

Calamintha nepeta s.l.

Campanula spec.

Carex spec. bicarpellat

Carex spec. tricarpellat

Caryophyllaceae

cf. Acer spec.

cf. Salix spec.

Chrysanthemum spec.

Cirsium spec.

Crepis spec.

Cyperaceae

Epilobium spec.

cf. Euphorbia spec.

Fabaceae (Leguminosae)

Lamiaceae

Malva spec.

Molinia arundinacea

Poa spec.

Poa pratensis/trivialis

Poaceae (Gramineae)

Rumex spec.

Silene spec.

Solanum spec.

Stachys spec.

Verbascum spec.

Veronica spec.

Indeterminata

Total uncarb.

\section{Carbonized plant remains}

cultivated plants

Anethum graveolens

Cerealia

Cerealia

Hordeum vulgare seed/fruit

chaff

grains

ear seed/fruit

seed/fruit

seed/fruit

seed/fruit

seed/fruit

seed/fruit

seed/fruit

seed/fruit

seed/fruit

seed/fruit

seed/fruit

seed/fruit

seed/fruit

seed/fruit

seed/fruit

seed/fruit

seed/fruit

seed/fruit

seed/fruit

seed/fruit

seed/fruit

seed/fruit

anther

seed/fruit

seed/fruit

seed/fruit

seed/fruit

seed/fruit

seed/fruit

seed/fruit

seed/fruit

seed/fruit

seed/fruit

seed/fruit

seed/fruit

seed/fruit

seed/fruit

seed/fruit

seed/fruit

seed/fruit

seed/fruit

seed/fruit

plant remains

samples
Lüscherz Lattrigen 'VI' Lattrigen 'VII'

$\begin{array}{cccc}\text { LUS } & \text { LAT } & \text { LAT0-3 } & \text { LAT0-3 } \\ 3400 & 3400 & 3200-3050 & 3200-3050 \\ 5 & 2 & 69 & 41 \\ 0.05 & 0.01 & 29.68 & 28.76\end{array}$

number number number presence all samples all samples all samples interval

samples

$\begin{array}{rrrrrr}2 & 5.3 & - & - & - & - \\ 1 & 5.3 & - & - & 1 & 2.4 \\ 244 & 63.2 & - & - & 24 & 17.0 \\ 2 & 5.3 & - & - & - & 2.4 \\ 1 & 5.3 & - & - & 1 & 7.3 \\ 14 & 10.5 & - & - & 5 & 7.3 \\ 751 & 84.2 & - & - & 3 & - \\ - & - & - & - & 4 & 9.7 \\ 2 & 5.3 & - & - & - & 75.6 \\ 6908 & 94.7 & - & - & 136 & - \\ 1 & 5.3 & - & - & - & - \\ 8 & 31.6 & - & - & - & -\end{array}$

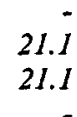


Site

Feature (cultural layer)

Dating (B.C.)

Number of samples

Total volume in litres
Nidau 'BKW'

$\begin{array}{cc}\text { NID5 } & \text { NID5 } \\ 3400 & 3400 \\ 30 & 30 \\ 24.52 & 23.57\end{array}$

number presence

all samples interval samples

Lüscherz Lattrigen 'VI'

Lattrigen 'VII'

$\begin{array}{cccc}\text { LUS } & \text { LAT } & \text { LAT0-3 } & \text { LAT0-3 } \\ 3400 & 3400 & 3200-3050 & 3200-3050 \\ 5 & 2 & 69 & 41 \\ 0.05 & 0.01 & 29.68 & 28.76\end{array}$

number number number presence all samples all samples all samples interval samples

\begin{tabular}{|c|c|c|c|c|c|c|c|}
\hline Hordeum vulgare & grains & 3765 & 84.2 & 1825 & 58 & 3185 & 58.5 \\
\hline Hordeum vulgare & rachis segments & 6 & 15.8 & - & - & 225 & 41.4 \\
\hline Linum usitatissimum & capsule segments & 8 & 21.1 & - & - & 9 & 14.6 \\
\hline Linum usitatissimum & seed/fruit & 31 & 47.4 & - & - & 139 & 48.7 \\
\hline Papaver somniferum & seed/fruit & 1 & 5.3 & - & - & 12 & 14.6 \\
\hline Pisum sativum & seed/fruit & 1 & 5.3 & - & - & 7 & 14.6 \\
\hline Triticum aestivum/durum & grains & 1042 & 68.4 & 2960 & 23 & 108 & 34.1 \\
\hline Triticum aestivum/durum & rachis segments & 6 & 15.8 & - & - & 18 & 26.8 \\
\hline Triticum dicoccon & spikelet forks & - & - & - & - & 104 & 46.3 \\
\hline Triticum dicoccon & grains & - & - & - & - & 586 & 60.9 \\
\hline Triticum monococcum & spikelet forks & - & - & - & - & 1 & 2.4 \\
\hline Triticum monococcum & grains & - & - & - & - & 4 & 4,8 \\
\hline Triticum monococcum/dicoccon & spikelet forks & 1 & 5.3 & - & - & - & - \\
\hline Triticum spec. & grains & 8 & 15.8 & 3 & & 359 & 29.2 \\
\hline Triticum spec. & rachis segments & - & - & - & - & 2 & 4.8 \\
\hline \multicolumn{8}{|l|}{ segetal weeds } \\
\hline Bromus secalinus & seed/fruit & - & - & - & - & 1 & 2.4 \\
\hline Vicia hirsuta & seed/fruit & - & - & - & - & 3 & 4.8 \\
\hline \multicolumn{8}{|l|}{ ruderal weeds } \\
\hline Sonchus asper & seed/fruit & - & - & - & - & 1 & 2.4 \\
\hline \multicolumn{8}{|l|}{ woodland glades, margin } \\
\hline Rubus fruticosus & seed/fruit & - & - & - & - & 1 & 2.4 \\
\hline Sambucus ebulus & seed/fruit & 2 & 10.5 & - & - & 1 & 2.4 \\
\hline \multicolumn{8}{|l|}{ woodland } \\
\hline Abies alba & needles & 3 & 10.5 & - & - & - & - \\
\hline Alnus glutinosa & seed/fruit & - & - & - & - & 6 & 7.3 \\
\hline Corylus avellana & seed/fruit & - & - & - & - & 1 & 2.4 \\
\hline Malus sylvestris & seed/fruit & 5 & 15.8 & - & - & 1 & 2.4 \\
\hline Quercus spec. & seed/fruit & 3 & 5.3 & - & - & 29 & 2.4 \\
\hline \multicolumn{8}{|l|}{ various } \\
\hline Fabaceae (Leguminosae) & seed/fruit & - & - & - & - & 2 & 4.8 \\
\hline Poa spec. & seed/fruit & - & - & - & - & 2 & 4.8 \\
\hline Poaceae (Gramineae) & seed/fruit & 4 & 5.3 & $=$ & - & - & - \\
\hline Indeterminata & plant remains & 10 & - & - & - & 2 & - \\
\hline Total carb. & & 4972 & - & 4788 & 81 & 4990 & - \\
\hline Total (carb. and uncarb.) & & 33941 & - & 4788 & 81 & 44760 & - \\
\hline
\end{tabular}

wheat is significantly less common (108 grains). Further, among the carbonized chaff remains, there are 104 spikelet forks from emmer and only 18 rachis segments from naked wheat. In contrast, in Nidau as in Lüscherz and Lattrigen ' $\mathrm{VI}$ ' there are no chaff remains from emmer.

Another glume-wheat species, einkorn (T. monococcum) was also found, but in insignificant amounts. The data are not therefore consistent with its active cul- tivation, rather with its incidental harvest with other cereals.

The cultivation of flax (Linum usitatissimum) was of importance throughout the second half of the 4th millennium B.C. Both seeds and capsule fragments of this plant occur frequently in most of the interval samples. The greater proportion of finds are uncarbonized (2975 capsule fragments and 6329 seeds); only a small fraction are carbonized (17 capsule fragments and 170 seeds). Its presence in the interval samples is in Nidau $90 \%$ and 


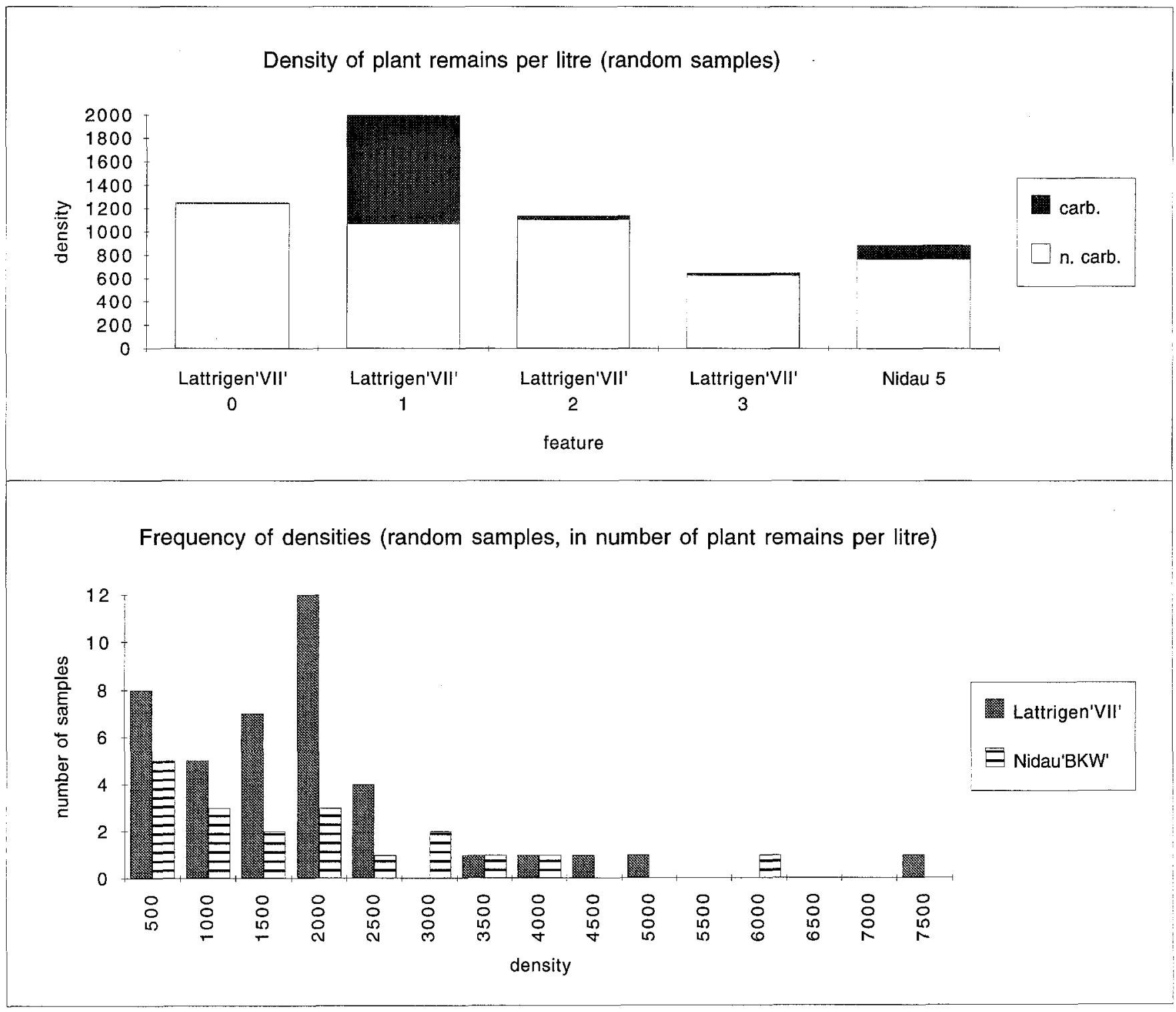

Fig. 3. Density of plant remains

$84 \%$, and in Lattrigen 'VII' $98 \%$ and $93 \%$ for uncarbonized seeds and capsule fragments, respectively. The respective densities were 65.9 and 41.3 items/l in Nidau and 159.7 and 65.7 items/l in Lattrigen 'VII'. Notably, there were also finds of specialized flax weeds such as Camelina sativa s.l. and Silene cretica. The latter species was frequently identified in sites of the Late Neolithic period in the northern Alpine foothills, but previously there were no archaeobotanical records of it after 2800 B.C. (Brombacher 1993).

An equally important cultivated plant was the opium poppy (Papaver somniferum). It occurs in the highest densities in the cultural layers from Lattrigen 'VII' (439 items per litre), where the presence of uncarbonized seeds also reached $100 \%$. Around 3400 B.C. (Nidau) the values are significantly lower (density $89 \mathrm{items} / \mathrm{l}$, but presence still $95 \%$ ), although this is probably due in part to the preservation conditions.

As the only legume, but also known from other Neolithic sites, a total of 9 peas (Pisum sativum; 1 uncarbonized, 8 carbonized) were identified.
Two probably cultivated plant species, dill (Anethum graveolens) and celery (Apium graveolens) were also found in Nidau and Lattrigen 'VII' with 8 and 5 finds respectively. Both have been recorded from other neolithic sites in Switzerland (Jacomet 1988), but always in insignificant amounts. While dill is indigenous to the eastern Mediterranean and west Asia (Zohary and Hopf 1993), wild forms of celery occur in the Mediterranean basin and in coastal regions of Europe (KörberGrohne 1987). The records from Swiss Neolithic lakeshore sites must be considered as introduction by humans and may represent a cultivation or an extensive use of these plants.

\section{Collected plants}

The numerous remains of collected edible plants clearly indicate that these plants played an important role in the subsistence pattern. In Nidau, berries are the most common: Fragaria vesca, Rubus idaeus and $R$. fruticosus, 
various Sambucus species and Physalis alkekengi. In Lattrigen 'VII', large amounts of Corylus avellana, Malus sylvestris and Quercus species were also found. Other species such a Prunus spinosa, Rosa spp., and Fagus sylvatica were exploited to a much lesser extent.

The highest presence (over 90\%) and also the most numerous finds were of $F$. vesca, $R$. fruticosus and $R$. idaeus. However, in terms of the calorific content, Corylus and Malus were more important.

For many other plant taxa, it is not possible to differentiate between cultivation, collection of useful plants or merely the presence of (uncollected) wild plants. It is most likely that Brassica rapa, Chenopodium album and Physalis alkekengi were used or collected. These three species are not very common at the Lake Biel sites, but were found in particularly high numbers at other Neolithic sites on Lake Zürich and Lake Constance (Bodensee) (Jacomet et al. 1989, 1991; Schlichtherle 1981). Valerianella dentata and Daucus carota were also possibly used.

\section{Agricultural activities}

What did the cultivated land look like that the cereals were grown in? Looking only at the carbonized weed diaspores that are found with the grain samples, no conclusion can be drawn because only a few carbonized wild plant seeds and fruits (Fabaceae and Poaceae) were found. The majority of wild plants that may have come from cultivated land are uncarbonized and thus interpretation is difficult because there are no Palaeobiocoenoses, (original combination of the growing plant community) only Thanatocoenoses (death assemblages, secondary combination of remains of taxa which did not necessarily grow in the same community) (Willerding 1991, 36). We cannot therefore rule out the possibility that the wild plant material was introduced into the cultural layer during or after its formation and does not originate from the same location. Further, the division of wild plants into ecological groups is particularly difficult in the plant habitats that have been heavily influenced by human activities, such as arable land and pasture, because the species combinations depend on the management practices of the time. During Neolithic times, many plants grew in cultivated fields which today are more common on the edges of woods, in clearings and in ruderal zones (Jacomet et al. 1989; Behre and Jacomet 1991). If we attempt nevertheless to interpret which species may have grown in the fields by drawing from weed rich cereal samples from other Neolithic sites (Piening 1981; Hopf 1968; Jacomet et al. 1989), the following can be seen.

Of the 16 taxa which are segetal today (after Ellenberg 1988), nine are winter weeds and seven summer weeds. Typical winter cereal weeds of today such as Agrostemma githago and Papaver argemone are absent. Instead, the species found belong to the Late Neolithic spectrum of weeds for the northern Alpine foothills. They include Fallopia convolvulus, Aphanes arvensis, Valerianella dentata, V. locusta and Viola tricolor which are not particularly tightly associated with winter

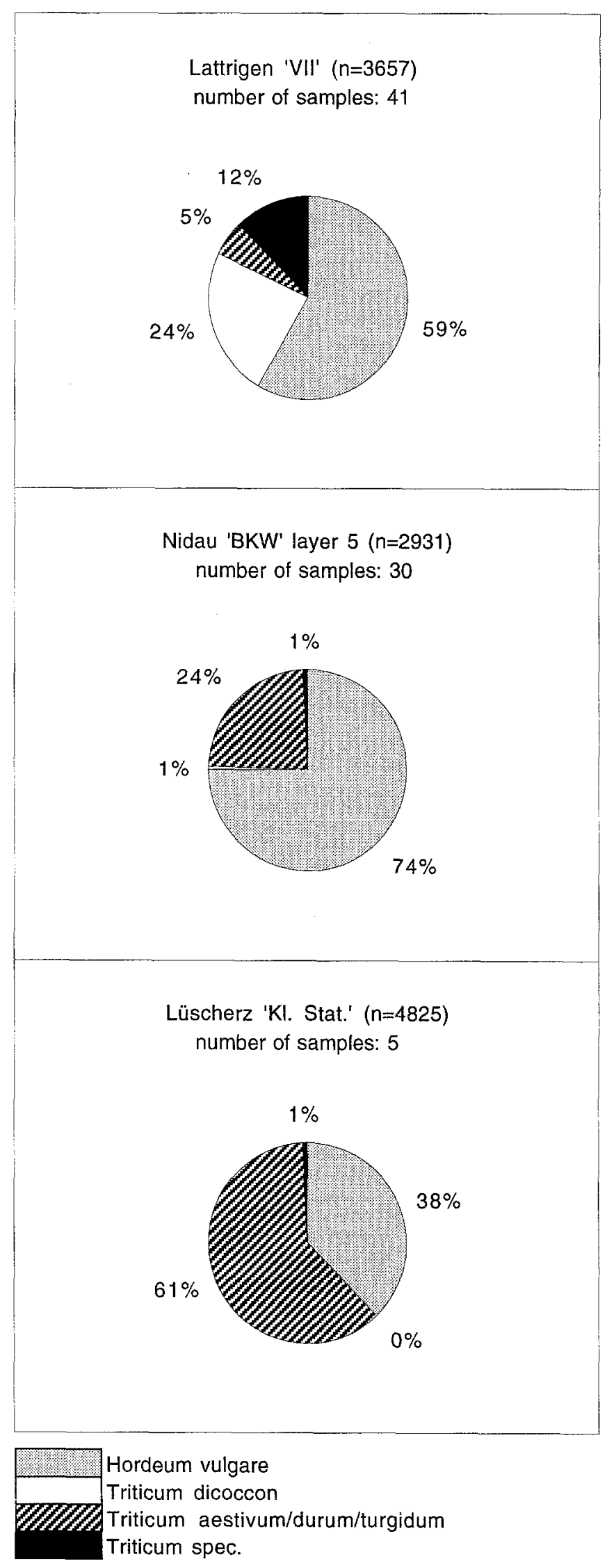

Fig. 4. Proportions of cereal grains 
cereal cultivation. The presence of most of these species was not above $60 \%$ at any site, with the exception of $F$. convolvulus which was found in $71 \%$ of the samples at Lattrigen 'VII'. A similar observation was made for the two flax weeds Silene cretica and Camelina sativa s.l. Both species are closely linked with flax cultivation, but are found at frequencies of only $32 \%$ and $11 \%$ in Nidau and $54 \%$ and $2 \%$ in Lattrigen 'VII', respectively. Among the other possible winter cereal weeds, Aphanes arvensis is most common, being found in $51 \%$ of samples. The other species occur at frequencies of less than $30 \%$. The summer weeds are also found at low frequencies; only Brassica rapa $(53 \%)$ in Nidau and Polygonum persicaria $(54 \%)$ and Stellaria media (49\%) in Lattrigen 'VII' were found at frequencies above $30 \%$. The rest (including Aethusa cynapium, Chenopodium polyspermum and Solanum nigrum) were only rarely found. In Lattrigen 'VII' the presence between samples as well as the actual numbers of remains of each species are significantly higher than in Nidau, where, except for Brassicarapa and Silene cretica, the frequencies were below 10\% (Table 1).

Most of the identified winter cereal weeds grow best on a slightly acidic, loamy clay soil. Weeds of more alkaline soils (Viola tricolor and Valerianella dentata) are in the minority. Similarly the summer cereal weeds indicate that the soil was slightly acidic, as is expected for the prevailing Würm moraine material. The cereal weeds cannot be considered in isolation from the ruderal plants. In the Neolithic age ruderal plants often grew on cultivated land, as shown by the diaspore evidence from grain store samples from various sites (Jacomet et al. 1989). The frequencies with which most of these plants were found was again lower in Nidau (around 3400 B.C.) than in Lattrigen 'VII' (3200-3000 B.C.). Considering the species list (Table 1), the dominant ones among the 27 species attributed to this group are from moist to average locations. The most common at Lattrigen 'VII' are Arctium minus (68\%), Chenopodium album (59\%), $R a$ nunculus repens $(76 \%)$ and Urtica dioica $(71 \%)$, though Lapsana communis, Polygonum aviculare and Sonchus asper are also often seen. In Nidau, Ranunculus repens was found at the highest presence, $42 \%$, all other species at less than $30 \%$.

The extremely low weed presence at Nidau indicates that there were no fields close to this site and that the grains found were brought from some distance. Similarly the rarity of carbonized and uncarbonized chaff remains here suggests that grain was not processed in large amounts at the site. Carbonized chaff was also rare in the samples from Lattrigen 'VII'; uncarbonized chaff remains were, however, found in large amounts (presence of Triticum dicoccon 46\%). The small number of uncarbonized chaff finds at Nidau could also be due to the rather poor preservation state of the cultural layer, but since carbonized remains, which would not be affected by the preservation state, are also rare, we conclude that no threshing floor was included in the Nidau site.

In summary, the spectrum of identified wild plants shows that for both Lattrigen 'VII' and Nidau the plant remains brought into the settlement originate in the surrounding area. Thisincludes a utilized area with a radius of around $1-3 \mathrm{~km}$.

\section{Crop cultivation during the Late Neolithic in Switzer- land}

Comparing the various Neolithic sites with wetland preservation in the Alpine foothills it becomes clear that crop plant species did not change during the Late Neolithic period. However, the prevalence of the different species varies with time and location. Generally, at least two different cereals were cultivated together with flax and poppy.

During the first half of the 3rd millennium B.C., the predominant cereals on the Swiss plateau were naked wheat (Triticum aestivum/durum/turgidum) and barley (Hordeum vulgare). Glume wheats ( $T$. dicoccon and $T$. monococcum) were unimportant at this time. Flax (Linum usitatissimum) and opium poppy (Papaver somniferum) also played a major role. The small numbers of peas (Pisum sativum) remains in the cultivated plant samples could be due to poor preservation and thus cannot reliably indicate the role of peas in this period.

The second half of the $3 \mathrm{rd}$ millennium is associated chiefly with changes in cereal cultivation. The importance of naked wheat, which was still predominant at Nidau, Lüscherz and Lattrigen 'VI' declined and was replaced by a glume wheat (usually emmer). The ascendancy of emmer took place between 3300-3200 B.C., but there are marked differences in its prevalence between sites. While naked wheat is unimportant in Lattrigen 'VII' and at Lake Zürich (Seefeld layer 3; Brombacher and Jacomet 1997) it still makes up a large proportion of the cereal remains at other sites (Twann: Ammann et al. 1981; Allensbach/Lake Constance: Karg 1990). Not until the time of the Corded Ware culture, after 2800 B.C., did naked wheat disappear almost completely from the settlements of the Alpine foothills.

There are no such strong differences in flax cultivation, although it is found in larger amounts after 3200 B.C. and especially from the beginning of the Corded Ware culture in east Switzerland and in the Saône-Rhône culture in west Switzerland. A different distribution is seen for the opium poppy which is found in greatest amounts in the remains of the Horgen culture at Lake Zürich (Jacomet et al. 1989) and at Lake Constance (Rösch 1990a). In western Switzerland, to which Lake Biel is also counted, poppy seems to have been much less important.

The developing preference for the less demanding glume wheat during the Late Neolithic could be connected with a more intensive cereal cultivation that, with the expansion of cultivated land and introduction of greater yield densities, led to a loss in soil quality. Evidence for this at Lake Constance is described by Rösch (1990a) and Billamboz (1990), and at Lake Zürich by Jacomet et al. (1989). The most intensive documented settlement of Lake Biel occurred in this period. Here too, there is evidence for a switch to more frugal cereal species together with the bringing of level ground into culti- 
Table 2. Crop cultivation during the Late Neolithic in Switzerland

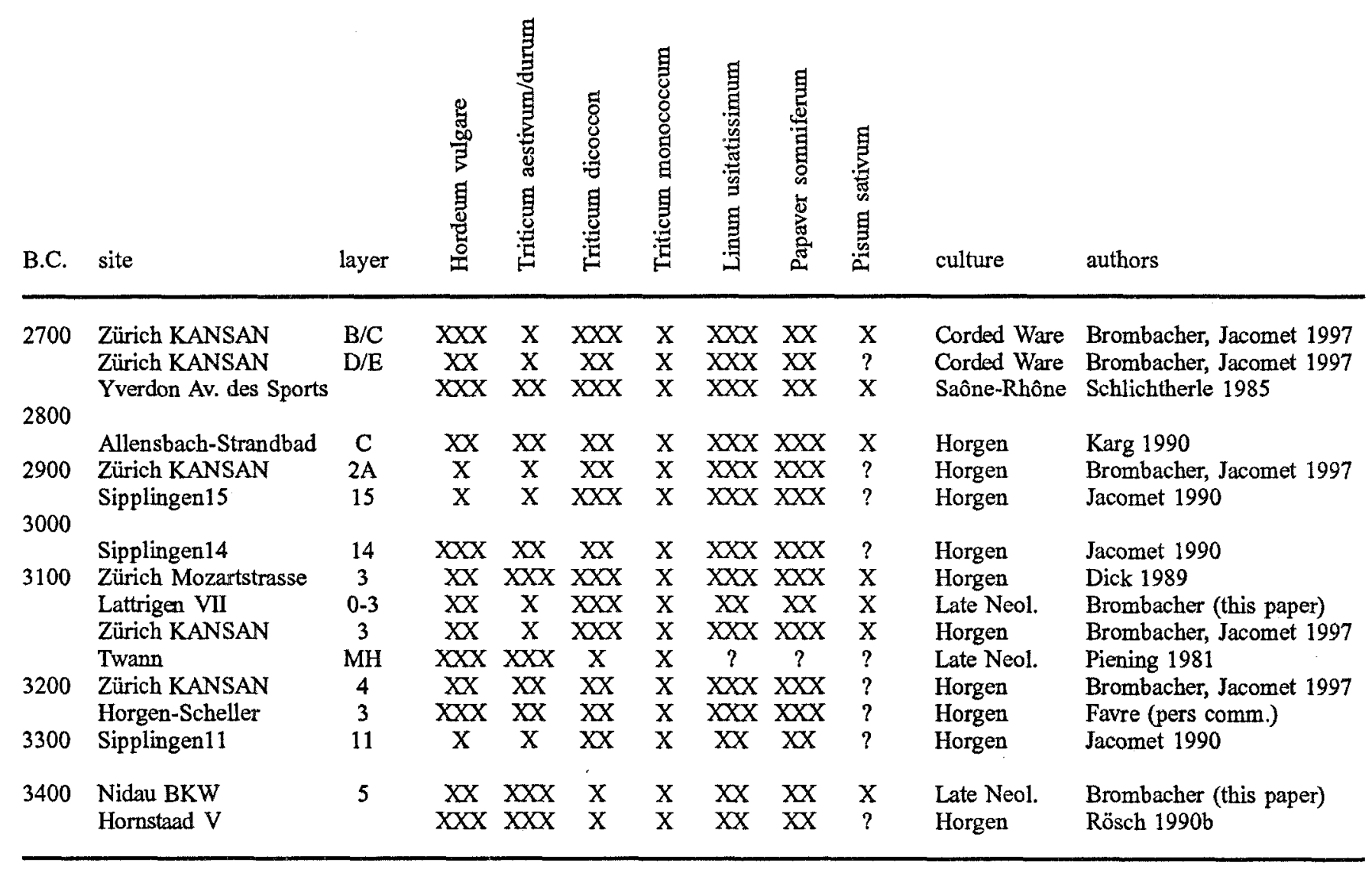

The importance of the various species is expressed by the number of crosses

Grain specimens are taken into consideration with first priority

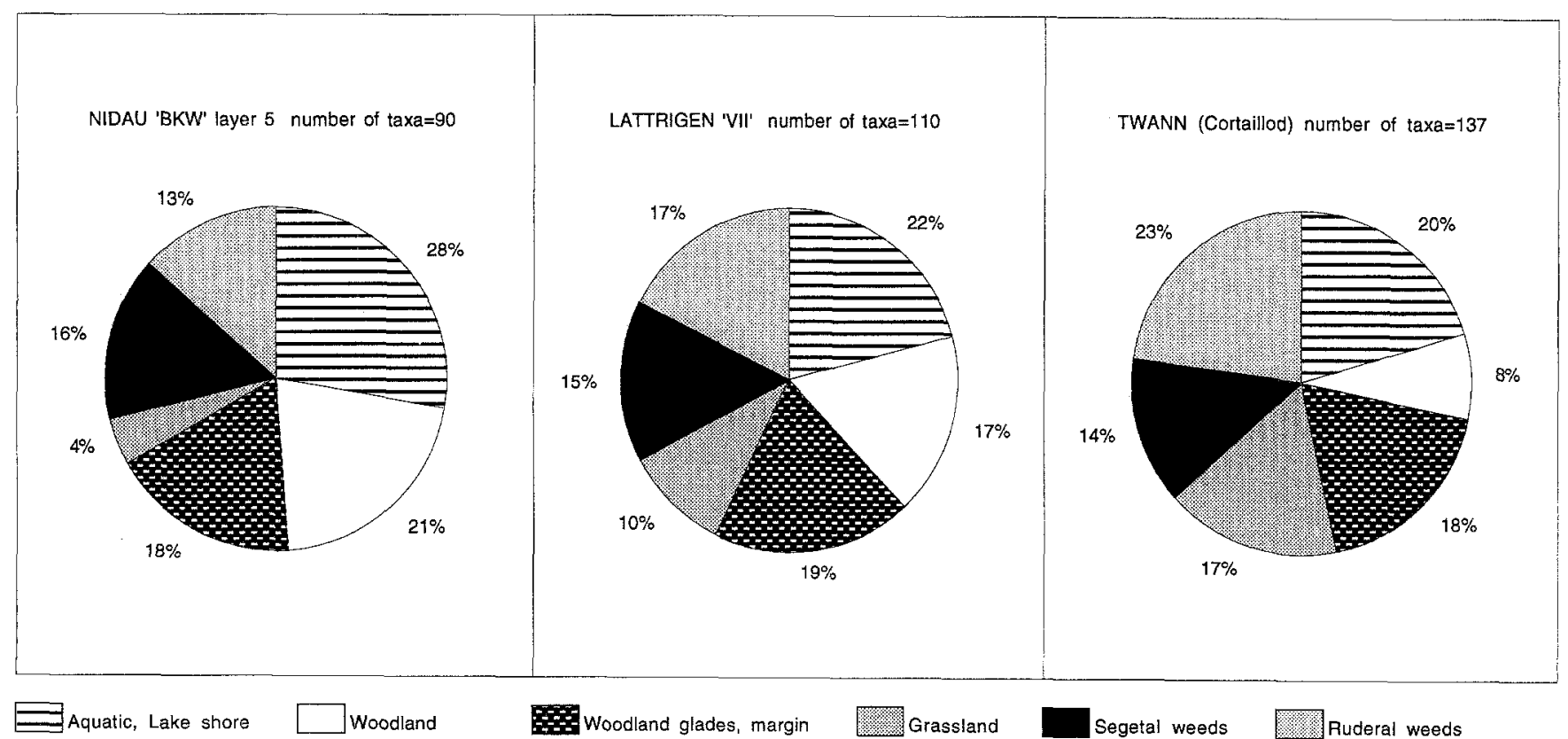

Fig. 5. Proportions of plant remains (seeds and fruits), arranged in ecological groups 
vation. Evidence for this derives from the weed flora: Jacomet et al. $(1989,1991)$ found more species typical of nutrient poor soil in this period such as Vicia tetrasperma, Euphorbia exigua and Trifolium arvense. At the lake Biel sites examined so far, remains of these weeds are rare with the exception of Twann. An intensification of glume wheat cultivation can, however, be clearly seen.

\section{Overview of the natural surroundings}

The different natural surroundings of the sites is clearly reflected in their plant spectra. Since there are only judgement samples from Lüscherz, the spectrum of Twann, a site on the left side of the lake (Cortaillod culture, Ammann et al. 1981) has been added. In Nidau, water, lake shore and forest plants reach up to $50 \%$ of all identified taxa, whereas in Lattrigen 'VII' only $39 \%$ and in Twann $28 \%$ belong to this group (Fig. 5). This difference clearly reflects that in Neolithic times, Nidau was in a riverbank area at the efflux of the lake. In contrast, only $20 \%$ of the taxa from Nidau are segetal weeds and grassland species, but $25 \%$ in Lattrigen 'VII' and $31 \%$ in
Twann. It is not surprising that Twann has the highest proportion of grassland species since natural open vegetation (arid grassland) occurs close to the site (Ammann et al. 1981).

\section{Lake shore vegetation}

A large portion of the identified plant remains originate from the immediate surroundings of the settlements, the lake shore and the alluvial zone. Water and lake shore plants, $28 \%$ of all taxa in Nidau and $22 \%$ in Lattrigen 'VII', make up the greatest proportion of species identified. In Nidau, this fraction is particularly large in samples from those parts of the cultural layer that were disturbed by the river. In Lattrigen 'VII', the flooded areas of the cultural layer have the highest proportion of water and lake shore plants, while the better preserved organic parts of the layer contain relatively few such remains. In Nidau oospores of stoneworts are very common, over 10000 specimens; but seeds and fruits of various Potamogeton spp., Myriophyllum spicatum and Ranunculus aquatilis were also found in large numbers. The lake was already at this time relatively nutrient rich as

Table 3. Lattrigen 'VII' and Nidau 'BKW'. Moss remains

Site

Feature

Ecological indicator value (after Ellenberg 1991)

\section{LattrigenVII Nidau BKW}

$0.3 \quad 5$

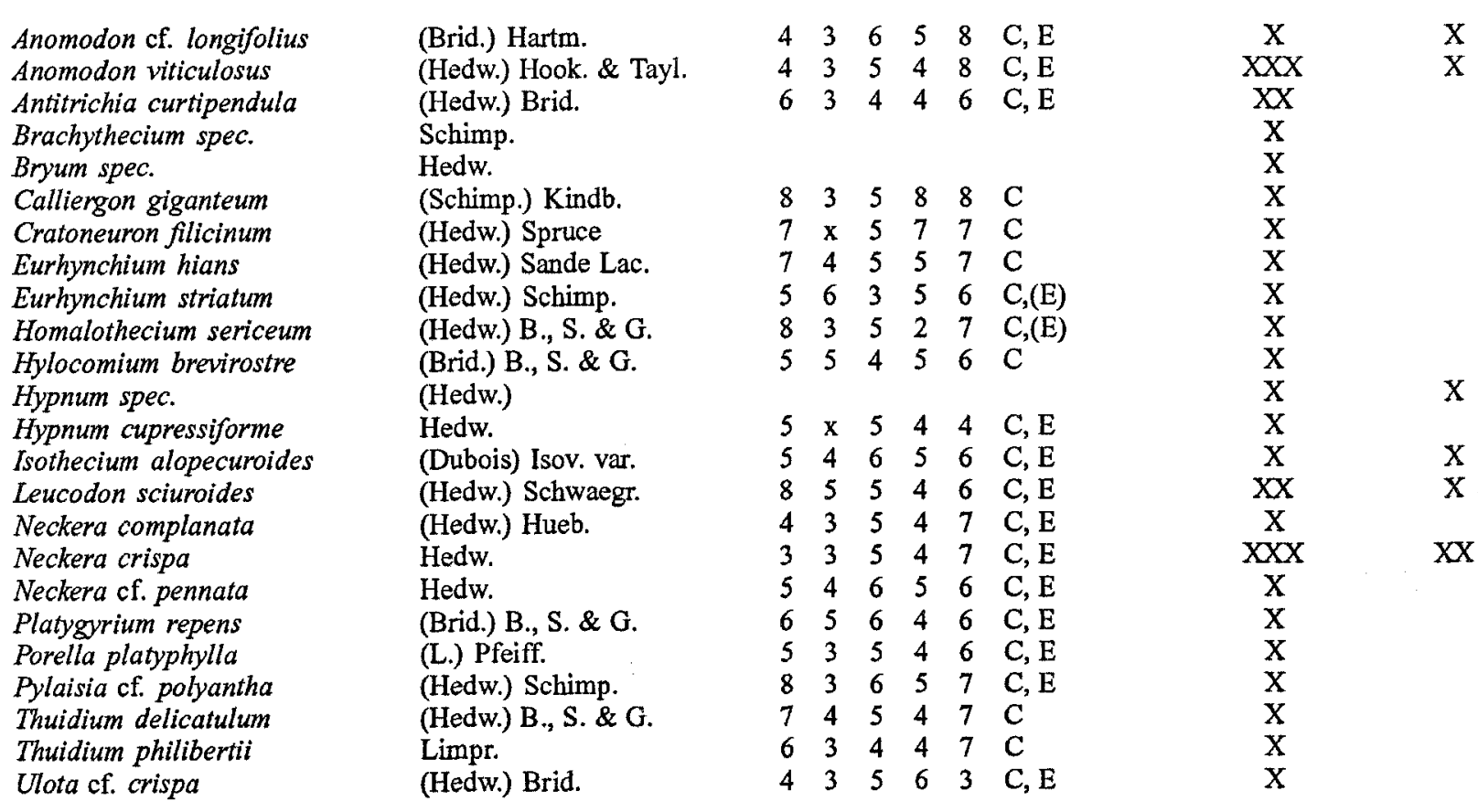

$\mathrm{C}=$-Chamaephyte, overwinters on the substrate, $\mathrm{E}=$ Epiphyte, growing on living plants (mostly trees)

$\mathrm{R}$ : indicator of mostly weakly acidic to weakly basic soils

F: mostly coolness indicator, only Calliergon indicates dampness

The importance of the various taxa is expressed by the number of crosses 


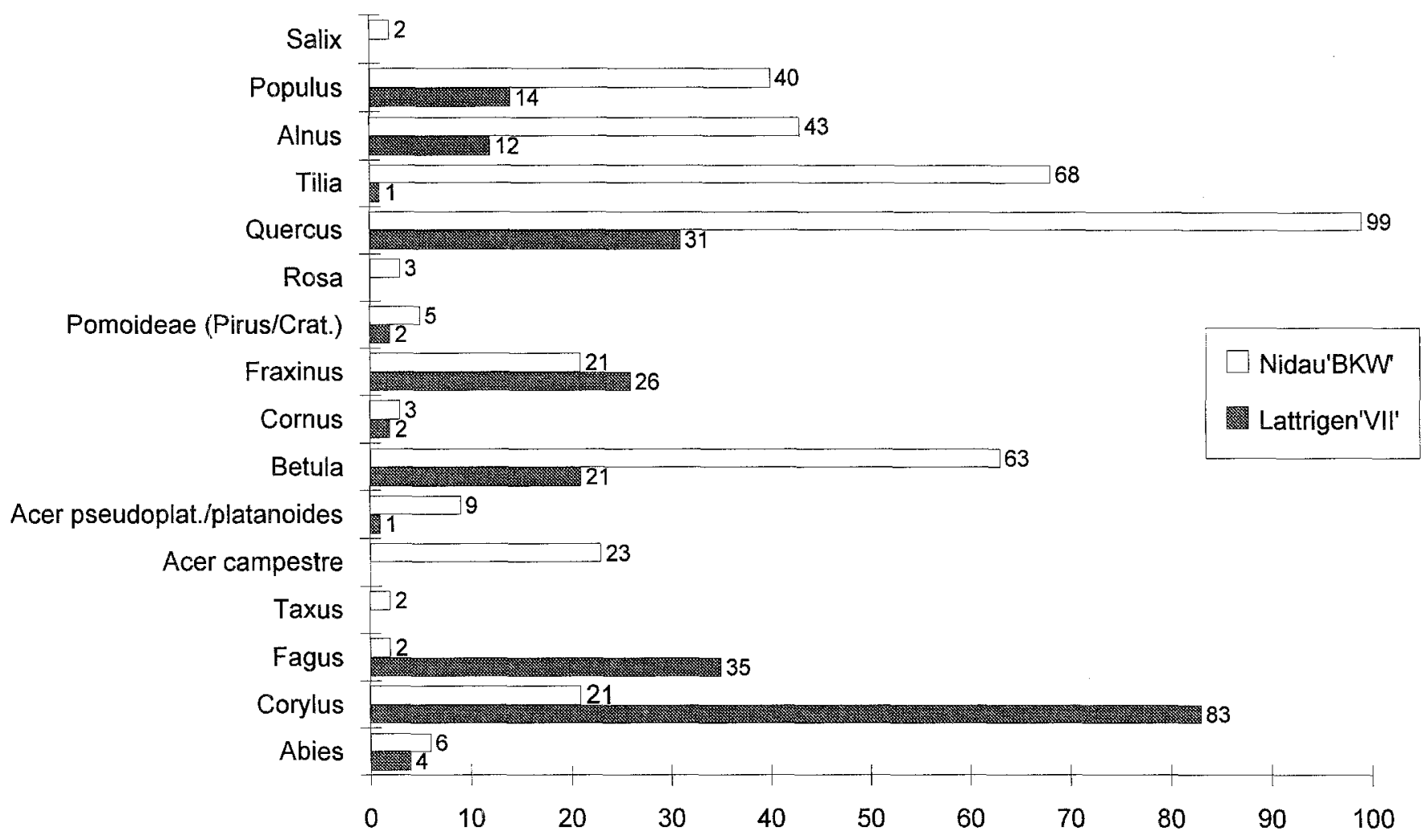

Fig. 6. Charcoal (number)

shown by the diaspore finds of Zannichellia palustris. Evidence of plants with floating leaves (Nuphar lutea and Nymphaea alba) indicate that the water was relatively calm close to the efflux. In Lattrigen 'VII' there are significantly fewer water plants: Najas marina is the most common, but Najas flexilis remains are also found in small numbers. On the basis of the frequent diaspore finds of reed-bed plants (Schoenoplectus lacustris and Phragmites australis) in Nidau we can conclude that at least close to the efflux there was an extensive reed bed around the lake. Plant remains from the large-sedge swamp (large numbers of Carex fruits) and from the neighbouring meadows with Molinia caerulea and Eleocharis palustris also occur regularly in many samples from Nidau and Lattrigen 'VII'. Of the rare species, we mention only the evidence of Corrigiola littoralis and Najas flexilis which are now extinct in the Swiss plateau. The first is a riverbank plant, which grows in sandpits and sandy riverbanks, the latter is an annual temperate water plant that is sensitive to temperature changes (Haas personal communication).

\section{Woodland}

Species typical of fen woods and flood-plain forest vegetation were also often identified. Diaspores from Alnus glutinosa and Frangula alnus reflect the alder swamp woods present at the time. Of the numerous identified taxa in the woods, woodland edges and clearings group, Clematis vitalba, Scrophularia nodosa, Prunus padus and Physalis alkekengi are frequently found in flood- plains. Utilization of the flood-plain forests is clearly demonstrated by the charcoal samples of which sub-samples were identified (five samples from Nidau, six samples from Lattrigen 'VII'; mesh fractions $\geq 4 \mathrm{~mm}$ ). A total of 800 pieces could be identified. The spectra of wood taxa at the two sites are shown in Fig. 6. In total 16 different taxa are represented (Lattrigen 'VII': 12, Nidau: 16). In Nidau taxa of river bank forests (Alnus, Populus, Salix) as well as Betula, Quercus and Tilia were prevalent. In Lattrigen 'VII' broadleaved woodland taxa (Corylus and Fagus) are more dominant among the charcoal samples.

The most common type of wood at Nidau is oak (Quercus) at $25 \%$ of the total. Oak was the most valuable building timber and so, despite its high energy content, we can suggest that it was not used as fuel in first priority. The oak charcoal is therefore mainly assumed to be building or carpentry waste or from a fire in the settlement. Other woods which were probably used as timber are white fir (Abies alba), ash (Fraxinus) and alder (Alnus). Lime (Tilia), birch (Betula) and poplar (Populus) appear frequently $(18,15$, and $10 \%$, respectively) in the charcoal spectrum from Nidau. Of the three, birch and poplar are most suitable for fuel, and lime was probably used for making bast. The spectrum from Lattrigen 'VII' is significantly different; hazel $(23 \%)$ and beech wood $(10 \%)$ are predominant, while oak is the third most common kind of charcoal identified at $9 \%$. The more valuable timber types such as maple (Acer) and yew (Taxus) are rare. Of note are the specimens of spruce cones (Picea abies) from Nidau since 
spruce cannot be expected to have grown as natural vegetation in the vicinity. These cones were presumably washed down from the nearest spruce stands in the Jura mountains in the Chasseral area $10 \mathrm{~km}$ away at ca. 1200 $1400 \mathrm{~m}$ asl. Regarding the whole wood spectrum, the presence of the different taxa correlates well with the probable vegetation in the environment of the settlements. This strongly suggests that the wood was felled as close as possible to the settlement.

\section{Grassland}

In contrast to Twann, where a large number of grassland plants were found as early as the first half of the 4th millennium B.C. (ca. 3840-3530 B.C.), relatively few grassland plants were found in the lakeshore settlements on the south bank of Lake Biel described here. In Lattrigen 'VII' the portion of plants belonging to the grassland group was only $10 \%$, in Nidau, just $4 \%$. The most common types (Ajuga reptans, Cerastium fontanum, Potentilla reptans, Prunella vulgaris and Stellaria graminea) all have a wide ecological amplitude and can also grow in woodland borders, paths, grazed fields and ruderal zones. Potentilla reptans and Prunella vulgaris indicate a rather wet or boggy ground. Evidence of moist meadows close to the shore is, however, infrequent; from these habitats only Molinia caerulea and Eleocharis palustris are found. The other taxa which could originate in grasslands are rare and chiefly found in the
Lattrigen 'VII' samples. This suggests that in the vicinity of both settlements there were no extensive grasslands. Evidence of calcareous swards, as found on the north bank of lake Biel at Twann (Ammann et al. 1981) and at the Auvernier-Brise-Lames site on the north shore of lake Neuchâtel (Baudais-Lundstrøm 1978), is except for a single seed specimen of Saponaria ocymoides not found at the sites described here.

\section{Mosses}

Mosses were identified in sub-samples taken from the $<2 \mathrm{~mm}$ material of many of the samples from Lattrigen 'VII' (22 samples) and from Nidau (4 samples). The aim of this study was to gain further palaeoecological information about the region surrounding the settlements through knowledge of the ecology of the species and to find which moss species were preferred by people. Recent classification and analyses of mosses from prehistoric settlements are rare (Rösch 1988, 1990c). Older evidence from Swiss lakeshore sites can be found in Neuweiler (1924).

Three hundred moss fragments were available and were assigned to 25 different taxa; 24 were mosses and one, Porella platyphylla, was a liverwort (Table 3). As in other Neolithic wetland sites, the two most common species were Anomodon viticulosus and Neckera crispa, being found in $95 \%$ and $82 \%$, respectively, of all the

Table 4. Measurements (in $\mathrm{mm}$ ) and indices of cereals $(\mathrm{N}=$ number of grains measured)

Taxon

mean values

standard deviation

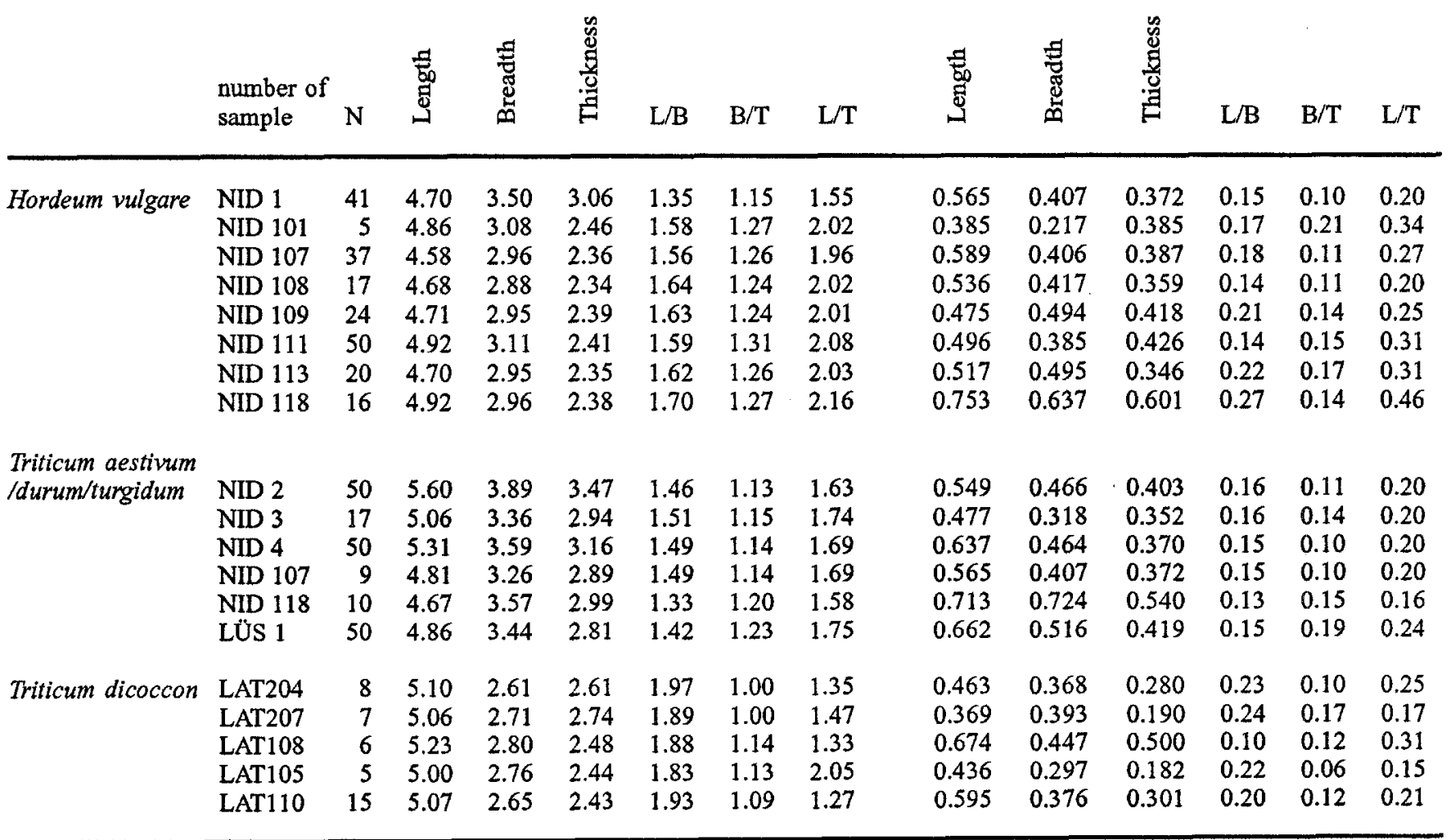



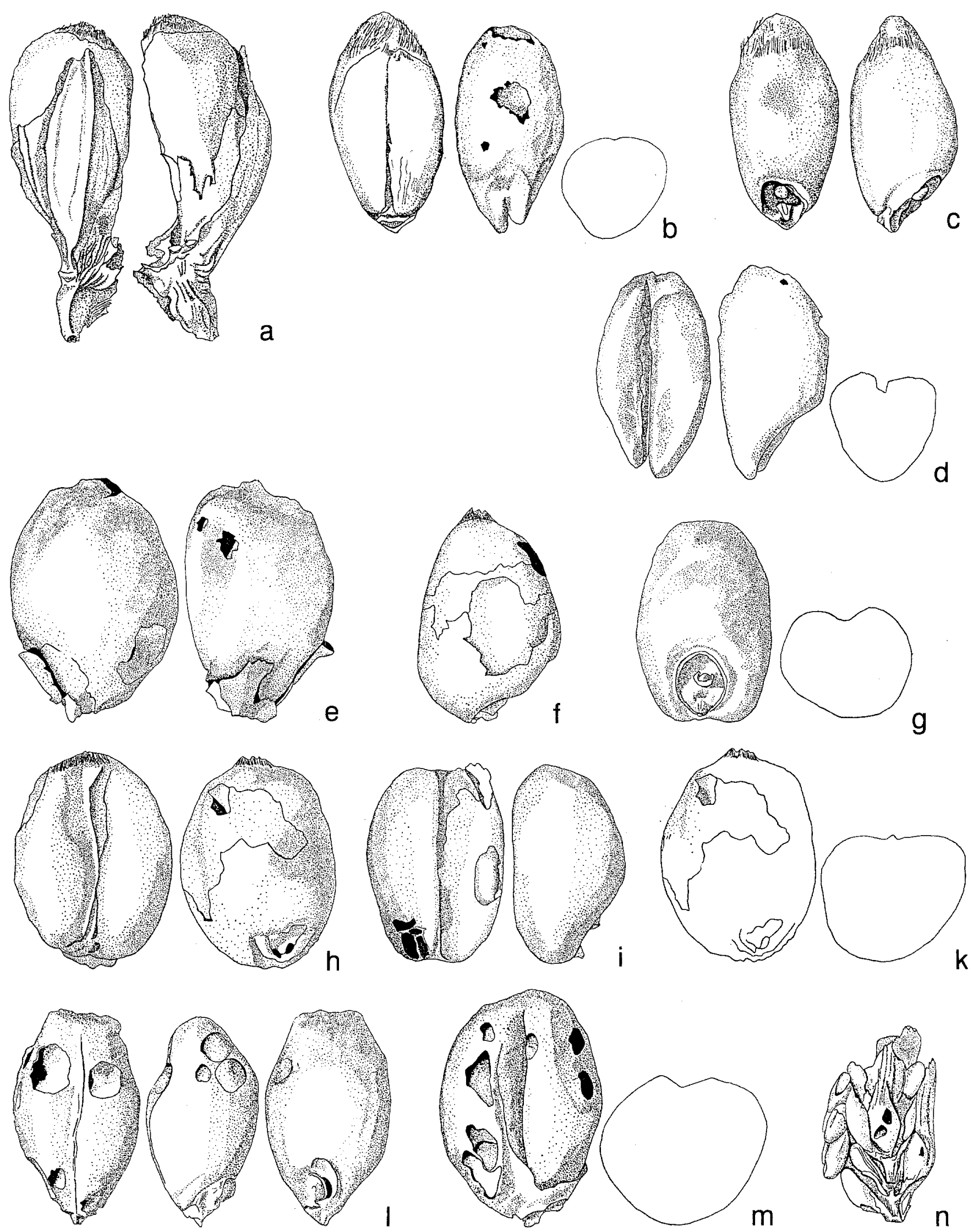

Fig. 7. Cereals (13 drawings): Various types of wheat grains: Triticum dicoccon (4, a-d), T. aestivum/durum/turgidum (6, e-k), Hordeum vulgare $(3,1-n)$ 

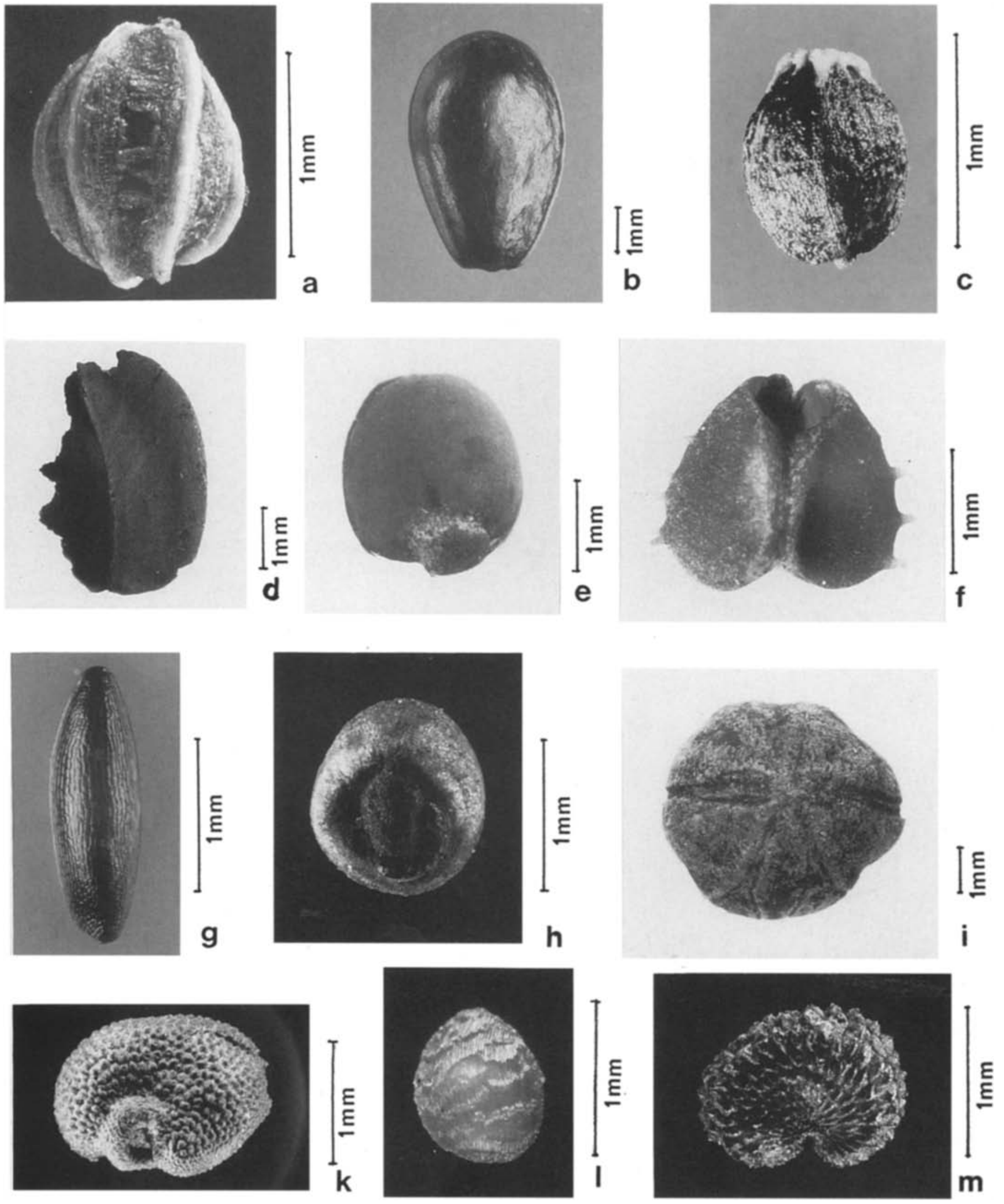

Fig. 8. Seeds and fruits (12 photos): a Apium graveolens, b Nuphar lutea, c Corrigiola litoralis, a Frangula alnus, e Humulus lupulus, $\mathbf{f}$ Myriophyllum spicatum, $\mathbf{g}$ Najas flexilis, h Teucrium scorodonia, 1 Cornus sanguinea, $\mathbf{k}$ Saponaria ocymoides, 1 Ranunculus aquatilis, m Silene cretica 

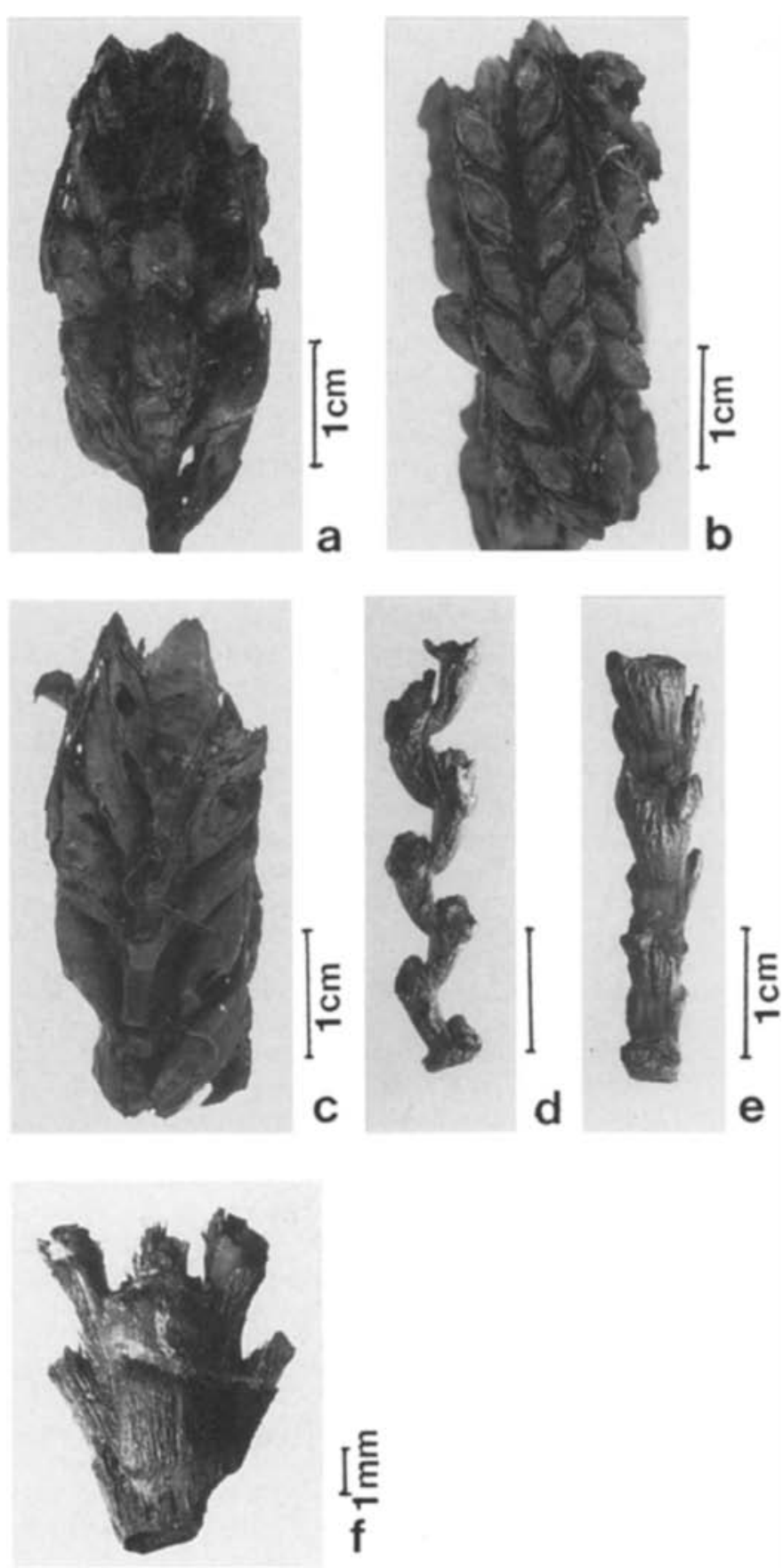

Fig. 9. Cereals ( 6 photos): Hordeum vulgare (3, a-c), Triticum aestivum/durum/turgidum $(3, \mathrm{~d}-\mathrm{f})$

samples examined. Antitrichia curtipendula and Leucodon sciuroides were also relatively common. These four moss species are all epiphytes which occurred on bark and sometimes on rocks in the Neolithic woods. All the other taxa were seldom found: dividing them according to habit, nine are epiphytes, four are ground mosses, eleven are found in broad habitat types and one, Calliergon giganteum, belongs to the bog and water mosses. The prevalence of the epiphytes shows that most of the mosses were gathered from or originated on tree trunks and branches in the nearby woods. Considering the ecological requirements of the different taxa, the predominant ones are the shadow loving bark mosses Neckera crispa and Anomodon viticulosus. Species normally found in open woodland e.g. Leucodon sciuroides and Antitrichia curtipendula were, however, also found. Among the ground mosses and broad habitat species, are some indicators of more open ground, such as Thuidium delicatulum which grows on unwooded ground.

\section{Comments on some of the identifications}

Wheat

Free threshing wheat, Triticum aestivum L./durum Desf./ turgidum L., was the most numerous wheat type in the samples. Of the 4133 carbonized grains found, 186 were measured. Most were of the dense-eared form (club wheat) with short, compact grains (Figs. 7, 9). This was also apparent from the L/B (length/breadth) index which in the grains in this group was 1.03-1.59 (Table 4). Jacomet (1987) defined the cut-off point between the long, slim grains of $T$. aestivum s. str. type and the short compact grains of compact-type as 1.5 . Only 38 grains had a L/B index of over 1.6 clearly above this value (1.6-1.85). Differentiation of tetraploid naked wheat of the $T$. durum/turgidum (macaroni/cone wheat) type from the $T$. aestivum (bread wheat) type is not possible from the grains. Rachis remains can indicate whether a wheat is tetraploid or hexaploid (Maier 1996), but were seldom found in the samples, however the swellings underneath the glume attachment and the straight course of the side edges fitted much better with tetraploid naked wheat (Fig. 9).

The second most common wheat was emmer ( $T$. dicoccon) which only occurred in the younger layers (site Lattrigen 'VII': 586 grains). Emmer grains are distinctly narrower but with $\mathrm{a} \mathrm{L} / \mathrm{B}$ index higher than that of the naked wheat grains. The values for the 41 grains measured lay between 1.55 and 2.41 (average 1.831.97). Many grains were deformed and no longer had distinguishing characteristics.

\section{Barley}

Barley was the most commonly identified grain at all of the sites examined. As well as the large number of grains (8833) 52 more or less highly fragmented ears were also found (Figs. 7, 9). These showed that this was a manyrowed barley. On several of these ear fragments the rachises, which bear the diagnostic characteristics distinguishing lax-eared (4 rowed) barley from dense-eared (6 rowed) barley, were visible. The rachis segments were very hairy which, according to Jacomet (1987), suggests they belong to dense-eared barley. The short length (1.7$2.7 \mathrm{~mm}$ ) and the L/B index of the rachis (2.5-3.1) are also consistent with dense-eared barley. These features can be clearly seen on the ear fragments (Fig. 9).

Acknowledgements. Sincere thanks are due to Peter J. Suter, Albert Hafner and Josef Winiger from the Archaeological Service of Canton Bern for providing the archaeological data and for taking botanical samples. I owe gratitude to Stefanie Jacomet (Basel) for critically reading the manuscript and for 
fruitful discussions. The drawings are by Marlies Klee (Basel) and the photographs of wild plants were taken by Georges Haldimann (La Chaux-de-Fonds), and of cereals by the Institute for Scientific Photography (Basel). Charcoal identification was performed by Angela Schlumbaum (Basel) and the mosses were identified by Josef Bertram (Allschwil/Basel). Financial support for the examination of plant remains was received from the Archaeological Service of Canton Bern.

\section{References}

Ammann B, Bollinger T, Jacomet-Engel S, Liese-Kleiber H and Piening U (1981) Die neolithische Ufersiedlungen von Twann, Band 14, Botanische Untersuchungen. Staatlicher Lehrmittelverlag, Bern

Ammann-Moser B (1975) Vegetationskundliche und pollenanalytische Untersuchungen auf dem Heidenweg im Bielersee. Beitr Geobot Landesaufn Schweiz 56

Baudais-Lundstrøm K (1978) Plant remains from a Swiss neolithic lake shore site: Brise-Lames, Auvernier. Ber Deutsch Bot Ges 91: $67-83$

Behre KE, Jacomet S (1991) The ecological interpretation of archaeobotanical data. In: Zeist $\mathrm{W}$ van, Wasylikowa $\mathrm{K}$ and Behre KE (eds), Progress in Old World Palaeoethnobotany. Balkema, Rotterdam, pp 81-108

Billamboz A (1990) Das Holz der Pfahlbausiedlungen Südwestdeutschlands, Jahrringanalysen aus archäodendrologischer Sicht. Ber RGK 71: 187-207

Bollinger T, Jacomet S (1981) Resultate der Samen- und Holzanalysen aus den Cortaillodschichten. In: Ammann B et. al., Die neolithischen Ufersiedlungen von Twann, Bd. 14, Botanische Untersuchungen

Brombacher C (1993) Prähistorische Nachweise der Kretischen Flachsnelke (Silene cretica L.) nördlich der Alpen. Diss Bot 196 (Festschrift Zoller): 491-498

Brombacher C, Jacomet S (1997) Ackerbau, Sammelwirtschaft und Umwelt: Ergebnisse archäobotanischer Untersuchungen. In: Schibler J et al., Ökonomie und Ökologie neolithischer und bronzezeitlicher Ufersiedlungen am Zürichsee. Monographien der Kantonsarchäologie Zürich 26

Dick M (1989) Wirtschaft und Umwelt cortaillod- und horgenzeitlicher Seeufersiedlungen in Zürich. Diss Bot 132

Ellenberg H (1988) Vegetation ecology of Central Europe. 4th ed. (tr. G.K. Strutt) Cambridge University Press, Cambridge

Ellenberg H (1991) Zeigerwerte der Gefäßpflanzen Mitteleuropas. Scripta Geobotanica 9. 2nd ed.

Hafner A (1996) Aspekte der Siedlungsarchäologie des Jung- und Spätneolithikums am Bielersee. In: Studien zum Siedlungswesen im Jungneolithikum. Beiträge zur Ur- und Frühgeschichte Mitteleuropas 10. Wilkau-Hasslau. pp 3-21

Hegg O (1980) Die heutige Pflanzenwelt der Region Biel. Jahrb Geogr Ges Bern 53: 43-70

Hegg O et al. (1993) Atlas schutzwürdiger Vegetationstypen der Schweiz. Bundesamt für Umwelt, Wald und Landschaft, Bern

Hopf M (1968) Früchte und Samen. In: Zürn H (ed.), Das jungsteinzeitliche Dorf Ehrenstein. Veröff Staatl Amt Denkmalpfl Stuttgart, Reihe A, Heft 10/II, pp 7-77

Jacomet S (1987) Prähistorische Getreidefunde. Eine Anleitung zum Bestimmen prähistorischer Weizen- und Gerstenfunde. Skript Botanisches Institut, Universität Basel

Jacomet S (1988) Pflanzen mediterraner Herkunft in neolithischen Seeufersiedlungen der Schweiz. In: Der prähistorische Mensch und seine Umwelt (Festschrift Körber-Grohne). Forsch u Ber Vor- Frühgesch Baden-Württemberg 31, pp 205-212

Jacomet S (1990) Veränderungen von Wirtschaft und Umwelt während des Spätneolithikums im westlichen Bodenseegebiet. In: Siedlungsarchäologie im Alpenvorland II. Forsch u Ber Vor- Frühgesch Baden-Württemberg 37, pp 295-324
Jacomet S et al. (1989) Archäobotanik am Zürichsee. Ber Zürcher Denkmalpfl Monogr 7

Jacomet S et al. (1991) Palaeoethnobotanical work on Swiss neolithic and bronze age lake dwellings over the past ten years. In: Renfrew J (ed), New light on early farming. Edinburgh University Press, Edinburgh, pp 257-276

Jones MK (1991) Sampling in palaeoethnobotany. In: Zeist W van, Wasylikowa K and Behre KE (eds), Progress in Old World Palaeoethnobotany. Balkema, Rotterdam, pp 53-62

Karg S (1990) Pflanzliche Grossreste der jungsteinzeitlichen Ufersiedlungen von Allensbach-Strandbad (Kr. Konstanz). In: Siedlungsarchäologie im Alpenvorland II. Forsch u Ber VorFrühgesch Baden-Württemberg 37, pp 113-165

Keller F (1854) Die keltischen Pfahlbauten in den Schweizerseen. Mitteilungen der Antiquarischen Gesellschaft Zürich 9, 3

Körber-Grohne U (1987) Nutzpflanzen in Deutschland. Theiss, Stuttgart

Maier U (1996) Morphological studies of free-threshing wheat ears from a Neolithic site southwest Germany, and the history of naked wheats. Veget Hist Archaeobot 5: 39-55

Neuweiler E (1924) Pflanzenreste aus den Pfahlbauten des ehemaligen Wauwilersees. Mitt Naturforsch Ges Luzern 9: 301-323

Piening U (1981) Die verkohlten Kulturpflanzenreste aus den Proben der Cortaillod- und Horgener Kultur. In: Ammann B et. al. (1981) Die neolithischen Ufersiedlungen von Twann. Bd. 14. Botanische Untersuchungen. Bern

Rösch M (1988) Subfossile Moosfunde aus prähistorischen Feuchtbodensiedlungen: Aussagemöglichkeiten zu Umwelt und Wirtschaft. In: Der prähistorische Mensch und seine Umwelt (Festschrift Körber-Grohne). Forsch u Ber Vor- Frühgesch Baden-Württemberg 31: 177-198

Rösch M (1990a) Veränderung von Wirtschaft und Umwelt während Neolithikum und Bronzezeit am Bodensee. Siedlungsarchäologische Untersuchungen im Alpenvorland. Ber RGK 71: $161-186$

Rösch M (1990b) Botanische Untersuchungen an Pfahlverzügen der endneolithischen Ufersiedlung Hornstaad-Hörnle V am Bodensee. In: Siedlungsarchäologie im Alpenvorland II. Forsch u Ber Vor- Frühgesch Baden-Württemberg 37: 325-351

Rösch M (1990c) Zur subfossilen Moosflora von AllensbachStrandbad. In: Siedlungsarchäologie im Alpenvorland II. Forsch u Ber Vor- Frühgesch Baden-Württemberg 37: 167-172

Schlichtherle H (1981) Cruciferen als Nutzpflanzen in neolithischen Ufersiedlungen Südwestdeutschlands und der Schweiz. Z Archäol 15: 113-124

Schlichtherle H (1985) Samen und Früchte. Konzentrationsdiagramme pflanzlicher Grossreste aus einer neolithischen Seeuferstratigraphie. In: Strahm C, Uerpmann HP (eds), Quantitative Untersuchungen an einem Profilsockel in Yverdon, Av. des Sports. Freiburg i.Br.

Veen M van der (1987) The plant remains. In: Heslop DH, The excavation of an Iron Settlement at Thorpe Thewles, Cleveland, 1980-1982. Counc Brit Archaeol Res Rep 65: 93-99

Willerding U (1991) Präsenz, Erhaltung und Repräsentanz von Pflanzenresten in archäologischem Fundgut. In: Zeist $W$ van, Wasylikowa $\mathrm{K}$ and Behre KE (eds), Progress in Old World Palaeoethnobotany. Balkema, Rotterdam, pp 25-51

Winiger J (1989) Bestandesaufnahme der Bielerseestationen als Grundlage demographischer Theoriebildung. Ufersiedlungen am Bielersee Bd. 1, Bern

Wohlfarth B, Schneider AM (1991) Late Glacial and Holocene Litho- and Biostratigraphy of Lake Biel, Western Switzerland. Bull Soc Vaud SC Nat 80: 435-457

Zohary D and Hopf M (1993) Domestication of plants in the Old World. 2nd ed. Clarendon Press, Oxford 